MODELING, IDENTIFICATION AND CONTROL, 1980, vol. 1, NO. 3, 135-163

doi:10.4173/mic.1980.3.1

\title{
A dynamic positioning system based on Kalman filtering and optimal control
}

\author{
JENS G. BALCHEN†, NILS A. JENSSEN‡, ELDAR MATHISEN§ \\ and STEINAR SALID†
}

Keywords: dynamic positioning, Kalman filtering, optimal control.

This paper describes a computer-based, dynamic positioning system for floating vessels. The system is based on a detailed mathematical model of vessel motion in response to forces from thrusters, wind, waves and water current. The system uses a Kalman filter for optimal estimation of vessel motions and environmental forces from wind, waves and current. The control system is based on feedback from the motion variables where the oscillatory, wave-induced component is removed by the estimator. Feedback from the water current estimate provides the integral action of the system and feed forward from the wind force estimates are implemented. Simulation results and recordings from actual operation of the system indicate an excellent system performance.

\section{Introduction}

The main purpose of a dynamic positioning system is to keep a floating vessel on a specified position by proper action of the propulsion system of the vessel. A dynamic positioning system, (a DP-system), includes one or more position and heading measurement systems, a set of control algorithms and a propulsion system. A DP-system is required in many offshore oil field operations such as drilling, pipelaying, diving support, etc.

A DP-system should be designed to keep the given vessel within specified position limits, with a minimum fuel consumption and with minimum wear and tear on the propulsion equipment. In addition, the DP-system should tolerate transient errors in the measurement system and also give an acceptable reaction in case of error in the propulsion system.

Dynamic positioning systems have been manufactured since the early sixties, mainly for drilling vessels and diving vessels. The traditional way of solving the control problem in a DP-system is by introduction of PID-controller for each of the surge, sway and yaw motions (Sargent and Cowgill 1976). This approach has several disadvantages. Due to couplings between the surge, sway and yaw motions, the integral action of the controller must be quite slow. A second disadvantage is due to the phase lag introduced in the control loops by the widely used notch filtering of the motion measurements. This is usually done because the first order wave forces acting on a floating structure may be extremely large and PD-systems are not intended to suppress wave introduced motions.

The known disadvantages of conventional DP-systems led the mechanical engineering and data technology company, Kongsberg Våpenfabrik $\mathrm{A} / \mathrm{S}$ of Norway, to

\footnotetext{
Received 2 July 1979

† University of Trondheim, The Norwegian Institute of Technology, Division of Engineering Cybernetics.

I SINTEF, The Foundation of Scientific and Industrial Research at The Norwegian Institute of Technology, Trondheim.

$\S$ A/S Kongsberg Vâpenfabrik, Kongsberg.
} 
initiate the development of a DP-system based on the concept of modern control theory, such as Kalman filtering and optimal control. The development of the estimation and control part of the DP-system was carried out in cooperation with SINTEF at the Norwegian Institute of Technology in Trondheim (Balchen et al. 1976).

The system was developed during the period 1975-1977. The first installation was carried out and tested during the summer of 1977 on board the vessel M/V Seaway Eagle of Seaway Offshore Services.

Since then, several systems have been installed and considerable experience in their operation has been gained. The rest of this paper deals with the design of the control and estimation part of the system. Several recordings from actual operation of the system are also given.

\section{Mathematical modelling}

The mathematical model is designed to be used in a Kalman filter for estimation of the motion variables of the vessel and essential environmental variables influencing the motion of the vessel. In order to separate the wave induced oscillatory part of the motion from the remaining part of the motion, the total vessel motion is modelled as the added outputs of a low-frequency model, (LF-model), and a high-frequency model, (HF-model). See Fig. 1.

\subsection{Coordinate systems}

It is convenient to apply two different coordinate systems for modelling purposes. An earth-fixed coordinate system is used to integrate the dynamic equations of motion. Another coordinate system, with the same origin as the earth-fixed system

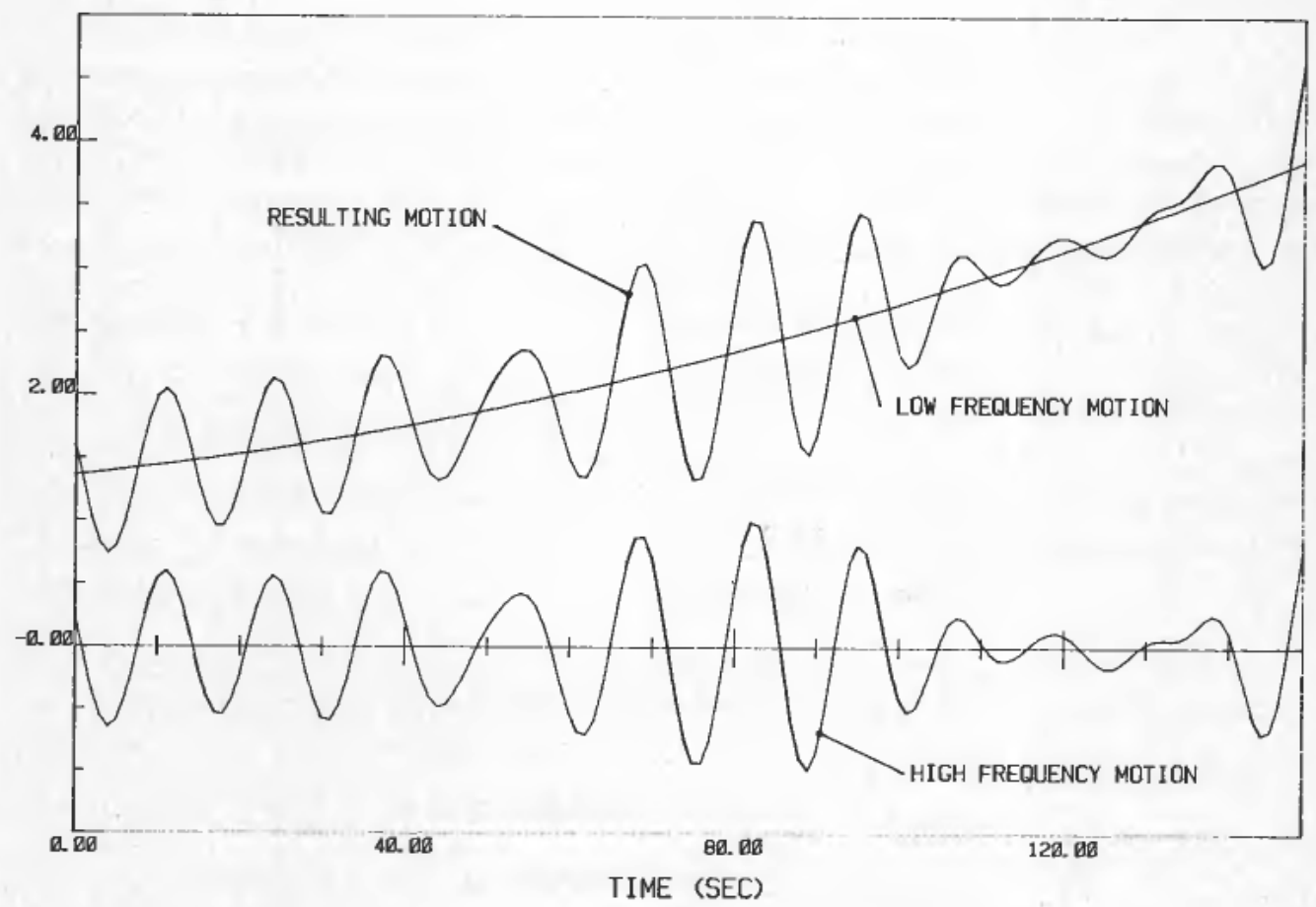

Figure 1. Low frequency and high frequency part of the vessel motion. 
and the axes parallel to the main axes of the vessel, is used for computing the forces acting on the vessel. For both systems, the $z$-axis is defined along the vertical, with the $x-y$ plane in the calm water surface. The coordinate system definitions are shown in Fig. 2.

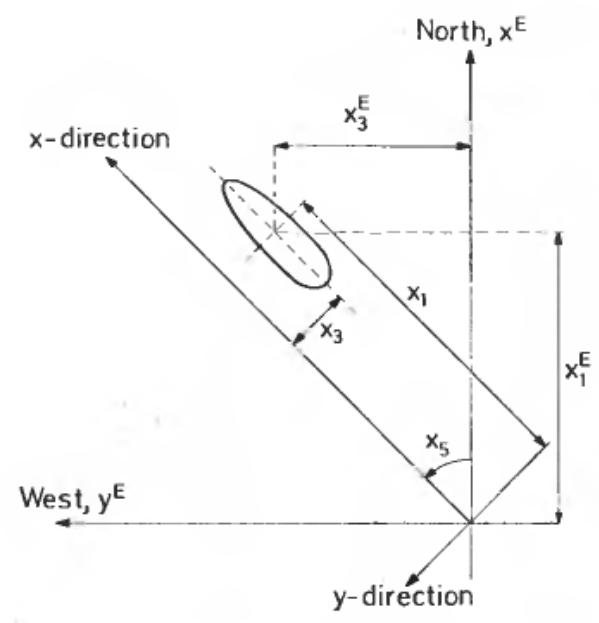

Figure 2. Definition of coordinate systems.

\subsection{The LF-model of vessel motion}

The low frequency model is designed to represent motions induced by wind, thrust and water current in the surge, sway and yaw coordinates. The model equations are formulated in the vessel parallel coordinate system. This coordinate system may be thought of as vessel parallel but 'frozen' during the force computations. For discrete time integration of the model equations, all variables are transformed to the vessel parallel coordinate system. The velocity and position increments are computed in this coordinate system and the resulting motion variables are transformed back to the earth-fixed coordinate system. This is repeated for each time increment of the integration.

The LF-model equations are

$$
\begin{aligned}
& \dot{x}_{\mathbf{L} 1}=x_{\mathbf{L} 2} \\
& \dot{x}_{\mathbf{L} 2}=-\frac{d_{1}}{m_{1}}\left|x_{\mathbf{L} 2}-x_{\mathrm{C} 1}\right|\left(x_{\mathbf{L} 2}-x_{\mathrm{C} 1}\right)+\frac{1}{m_{1}}\left(u_{1}+v_{1}\right)+\eta_{\mathbf{L} 1} \\
& \dot{x}_{\mathbf{L} 3}=x_{\mathbf{L} 4} \\
& \dot{x}_{\mathbf{L} 4}=-\frac{d_{2}}{m_{2}}\left|x_{\mathbf{L} 4}-x_{\mathrm{C} 2}\right|\left(x_{\mathbf{L} 4}-x_{\mathrm{C} 2}\right)+\frac{1}{m_{2}}\left(u_{2}+v_{2}\right)+\eta_{\mathbf{L} 2} \\
& \dot{x}_{\mathbf{L} 5}=x_{\mathbf{L} 6} \\
& \dot{x}_{\mathbf{L} 6}=-\frac{d_{3}}{m_{3}}\left|x_{\mathbf{L} 6}\right| x_{\mathbf{L} 6}-\frac{d_{4}}{m_{3}}\left|x_{\mathbf{L} 4}-x_{\mathrm{C} 2}\right|\left(x_{\mathbf{L} 4}-x_{\mathrm{C} 2}\right)+\frac{1}{m_{3}}\left(u_{3}+v_{3}+x_{\mathrm{C} 3}\right)+\eta_{\mathbf{L} 3}
\end{aligned}
$$

where the following definitions are used 


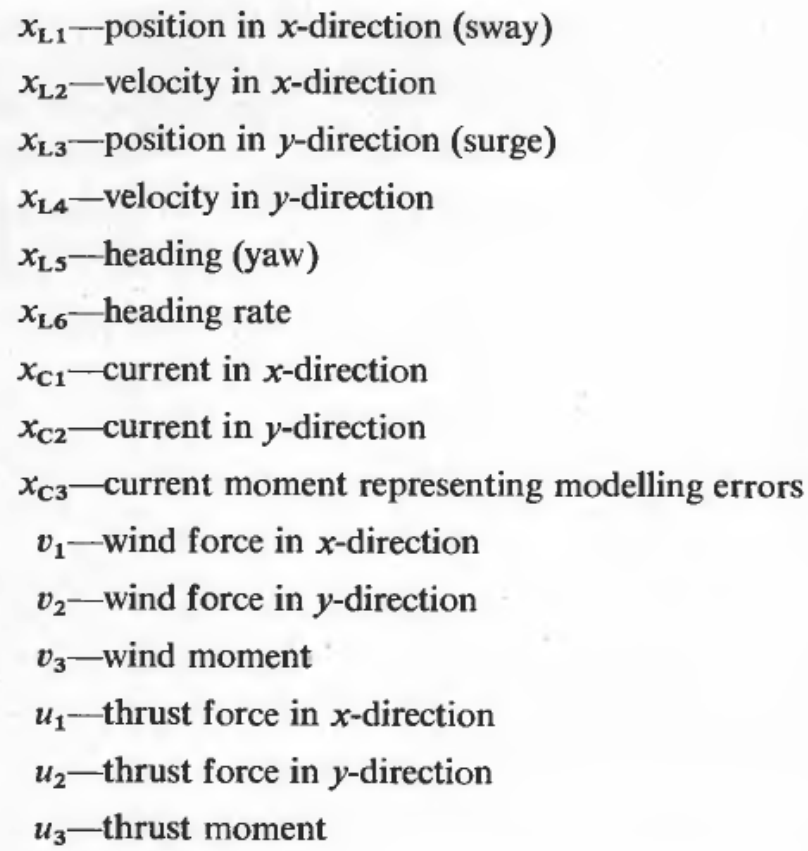

$\eta_{\mathrm{L} i}, i=1,2,3$, are assumed to be zero mean gaussian white noise processes and $d_{i}$, $i=1,2,3,4$, are drag and momentum coefficients. These are generally given as functions of the difference between the heading angle of the vessel and the water current direction. $m_{i}, i=1,2,3$, are inertial coefficients which are assumed to be constants.

\subsection{The HF-model of vessel motion}

The high frequency model is formulated in the vessel parallel coordinate system. The surge, sway and yaw motions are modelled by three separate harmonic oscillators with a variable frequency and with white process noise inputs representing modelling errors and unpredictable wave noise. The mathematical formulation for the HFmotion becomes:

$$
\begin{aligned}
& \dot{x}_{\mathrm{H} 1}=x_{\mathrm{H} 2} \\
& \dot{x}_{\mathrm{H} 2}=-\omega_{1}{ }^{2} x_{\mathrm{H} 1}+\eta_{\mathrm{H} 1} \\
& \dot{x}_{\mathrm{H} 3}=x_{\mathrm{H} 4} \\
& \dot{x}_{\mathrm{H} 4}=-\omega_{2}{ }^{2} x_{\mathrm{H} 3}+\eta_{\mathrm{H} 2} \\
& \dot{x}_{\mathrm{H} 5}={ }_{\mathrm{H}} x_{6} \\
& \dot{x}_{\mathrm{H} 6}=-\omega_{3}{ }^{2} x_{\mathrm{H} 5}+\eta_{\mathrm{H} 3} \\
& \dot{\omega}_{1}=\eta_{\mathrm{H} 4} \\
& \dot{\omega}_{2}=\eta_{\mathrm{H} 5} \\
& \dot{\omega}_{3}=\eta_{\mathrm{H} 6}
\end{aligned}
$$


where

$$
\begin{aligned}
& x_{\mathrm{H} 1}-\text { HF-part of position in } x \text {-direction (surge) } \\
& x_{\mathrm{H} 2}-\text { HF-part of surge rate } \\
& x_{\mathrm{H} 3}-\text { HF-part of position in } y \text {-direction (sway) } \\
& x_{\mathrm{H} 4}-\text { HF-part of sway rate } \\
& x_{\mathrm{H} 5}-\text { HF-part of yaw } \\
& x_{\mathrm{H} 6}-\text { HF-part of yaw rate } \\
& \omega_{1}-\text { angular frequency of HF-motion in surge } \\
& \omega_{2} \text {-angular frequency of HF-motion in sway } \\
& \omega_{3} \text {-angular frequency of HF-motion in yaw }
\end{aligned}
$$

and where $\eta_{\mathbf{H}}$ is assumed to be zero mean, gaussian white noise process $(i=1,2$, ..., 6).

\subsection{Modelling of environmental disturbance}

The water current model includes the current velocity components in earth fixed coordinates. The current components are modelled as slowly varying parameters by

where

$$
\begin{aligned}
\dot{x}_{\mathrm{C} 1} & =\eta_{\mathrm{C} 1} \\
\dot{x}_{\mathrm{C} 2} & =\eta_{\mathrm{C} 2} \\
\dot{x}_{\mathrm{C} 3} & =\eta_{\mathrm{C} 3}
\end{aligned}
$$

$$
\eta_{\mathrm{C} i}-\text { zero mean white noise processes }(i=1,2,3)
$$

The current vector applied to eqns. (2), (4) and (6) are derived through the rotational transform matrix

$$
A=\left[\begin{array}{cc}
\cos x_{5} & -\sin x_{5} \\
\sin x_{5} & \cos x_{5}
\end{array}\right]
$$

by

$$
\left[\begin{array}{l}
x_{\mathrm{C} 1} \\
x_{\mathrm{C} 2}
\end{array}\right]=A^{\mathrm{T}}\left[\begin{array}{l}
x_{\mathrm{C} 1}{ }^{\mathrm{E}} \\
x_{\mathrm{C} 2}{ }^{\mathrm{E}}
\end{array}\right]
$$

where $x_{5}$ is the sum of the LF- and HF-parts of the yaw angle.

The wind speed and direction are modelled as a sum of their slowly varying mean value parts and their rapidly varying parts, (wind gusts). Hence, we write the wind model as

$$
\begin{aligned}
& \dot{x}_{\mathrm{w} 1}=\eta_{\mathrm{w} 1} \\
& \dot{x}_{\mathrm{w} 2}=a_{1} x_{\mathrm{w} 2}+\eta_{\mathrm{w} 2} \\
& \dot{x}_{\mathrm{w} 3}=\eta_{\mathrm{w} 3} \\
& \dot{x}_{\mathrm{w} 4}=a_{2} x_{\mathrm{w}_{4}}+\eta_{\mathrm{w} 4}
\end{aligned}
$$


where

$x_{\mathrm{w}_{1}}-$ slowly varying part of the wind speed

$x_{\mathrm{W}_{2}}-$ rapidly varying part of the wind speed

$x_{\mathrm{w}_{3}}-$ slowly varying part of the wind direction

$x_{\mathrm{W} 4}$-rapidly varying part of the wind direction

$\eta_{\mathrm{w} i}$-zero mean white noise gaussian process $(i=1,2,3,4)$

$a_{i}$-constant coefficients $(i=1,2)$

The wind forces $v_{t}, i=1,2,3$, defined in the LF-model of the vessel motion is then given by

$$
v_{i}=f_{i}(\beta)\left(x_{\mathrm{w}_{1}}+x_{\mathrm{w}_{2}}\right)^{2}
$$

where $f_{i}$ are wind drag and momentum coefficients. These are functions of $\beta$, the angle between the wind direction and the vessel heading given by

$$
\beta=x_{w_{3}}+x_{w_{4}}-x_{5}
$$

\subsection{Modelling of the thruster system}

The resulting thrust vector is computed by adding the effect of each thruster. The force generated by each thruster is computed as a function of speed, azimuthand pitch-angle in a rather complicated way. The computed thrust force is also modelled as a function of the relative velocity between the water and the thrusters.

\subsection{The measurement model}

Position measurements may be carried out by means of several types of sensors, most commonly used are hydroacoustic position reference systems, taut wire systems and short range radio navigation systems. The heading measurement is given by a gyrocompass. We will assume that the position measurements are given in the vessel parallel coordinate system $(x, y)$. For notational simplicity, we assume that only one position measurement system is active. The position and heading measurement model is then given by

where

$$
\begin{aligned}
& y_{1}=x_{\mathrm{L} 1}+x_{\mathrm{H} 1}+w_{1} \\
& y_{2}=x_{\mathrm{L} 3}+x_{\mathrm{H} 3}+w_{3} \\
& y_{3}=x_{\mathrm{L} 5}+x_{\mathrm{H} 5}+w_{3}
\end{aligned}
$$

$y_{1}$-position measurement in $x$-direction

$y_{2}$-position measurement in $y$-direction

$y_{3}$-heading measurement

$w_{t}-$-zero mean independent white noise processes $(i=1,2,3)$

The wind speed and direction measurements are modelled by

$$
\begin{aligned}
& y_{w_{1}}=x_{w_{1}}+x_{\mathrm{w}_{2}}+w_{\mathrm{w}_{1}} \\
& y_{\mathrm{w}_{2}}=x_{\mathrm{w}_{3}}+x_{\mathrm{w}_{4}}-x_{\mathrm{L} 5}-x_{\mathrm{H} 5}+w_{\mathrm{w}_{2}}
\end{aligned}
$$


where

$$
\begin{aligned}
& y_{\mathrm{w}_{1}}-\text { wind speed measurement } \\
& y_{\mathrm{w}_{2}}-\text {-measurement of wind direction relative to the } x \text {-axis } \\
& w_{\mathrm{w}_{i}}-\text { zero mean independent white noise processes }(i=1,2) .
\end{aligned}
$$

\section{Estimator design}

Based on the previous stochastic model of the environment, the vessel and the thruster system, a complete extended Kalman filter, (Jazwinski 1970), could be designed. However, the heavy computational load imposed by the extended Kalman filter equations may be greatly decreased by introducing the following approximations:

- The estimation of the wind states and the estimation of the vessel motion are decoupled. By this, we mean that the wind state estimates are updated by using the wind measurements only and that the estimation error of the wind force acting on the vessel is included in the process noise terms $\eta_{\mathrm{L} i}$ and $\eta_{\mathrm{Hi}}$. It is also assumed that the uncertainty in the vessel heading is included in the measurement noise of the wind direction measurement eqn. (30).

-A steady state filter gain matrix is used in the wind filter.

-The estimation of the surge-, sway- and yaw-motions of the vessel are decoupled, in the sense that the prediction error in one of the measured coordinates is not used for updating the position and velocity in the two other coordinates. The couplings in the prediction model of the vessel motion are, of course, included.

- Because the vessel dynamics do not influence the water current and because the current variations are slow, the current estimates are updated by the prediction errors of surge and sway measurements transformed to north oriented coordinates and the current moment variable is updated by the yaw measurement prediction error. The filter gain matrix for the current variables is assumed to be constant.

The resulting filter structure is shown in Fig. 3, where $K_{\mathrm{SU}}, K_{\mathrm{Sw}}, K_{\mathrm{YA}}, K_{\mathrm{w}}$ and $K_{\mathrm{C}}$ are the filter gain matrices for the submodels of surge, sway, yaw, wind and water current, respectively. Note that the surge and sway models are integrated in earth fixed coordinates and updated in vessel parallel coordinates. We shall now discuss the different subfilters.

\subsection{The surge, sway and yaw filters}

Initial experiments with extended Kalman filtering based on full covariance computations showed the following:

-After a short initial period, the filter gains for estimation of positions and velocities were constants except for very small variations, (smaller than $\sim 2-3 \%$ ).

- The filter gains for estimation of the HF-motion frequencies oscillate with the same frequency as the HF-motion and with zero mean value.

In order to reduce the computational load imposed by the filtering algorithms, the filter gain coefficients for updating of positions and velocities are assumed to be constants. However, this is impossible for the frequency updating part of the filter 


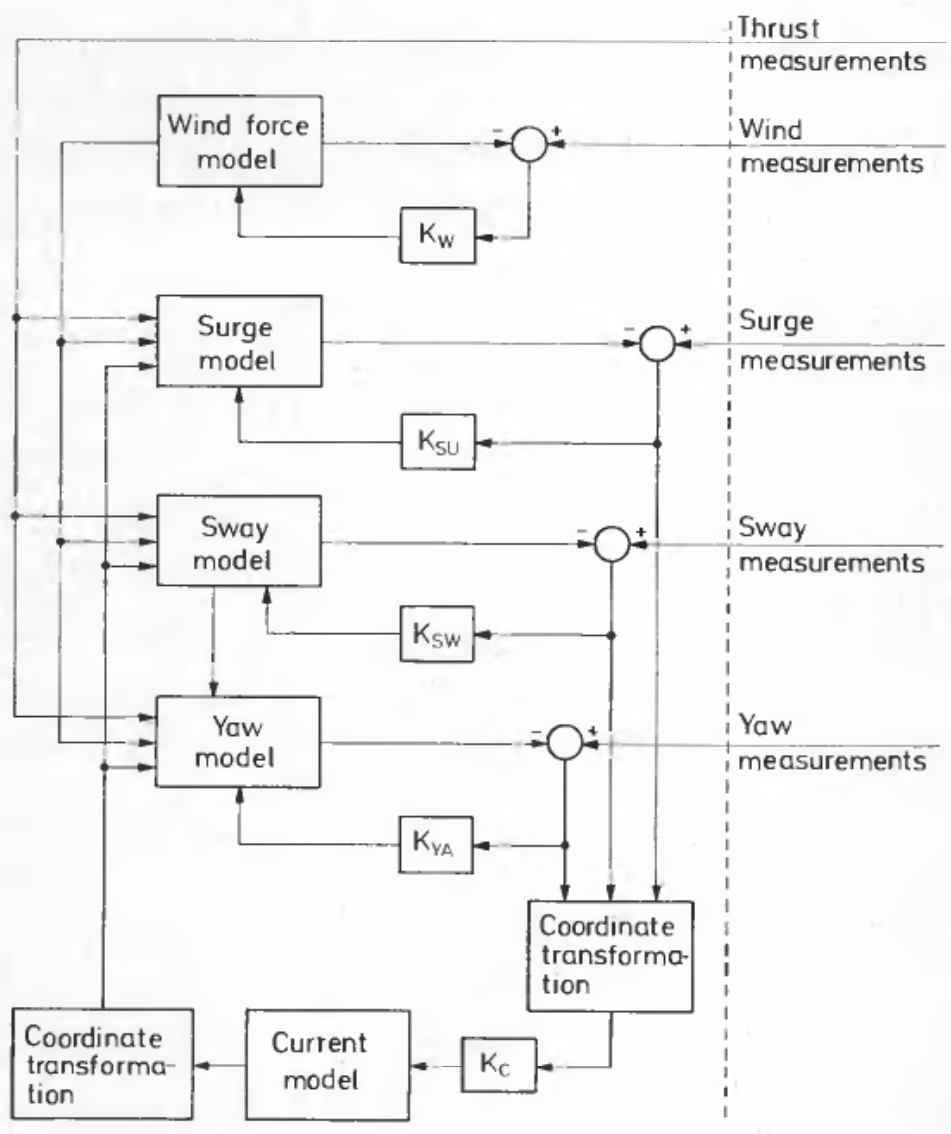

Figure 3. The filter structure. (Note that the surge and sway models are integrated in earthfixed coordinates. This is not shown in the figure).

gain matrix. We shall show that this part of the filter gain matrix can be assumed to be a linear combination of the high frequency position and velocity estimates.

Let us consider the surge coordinate. If the oscillation frequency, $\omega_{1}$, of the HFmotion is assumed to be known, the approximate surge filter is given by

$$
\begin{aligned}
& \hat{x}_{\mathrm{L}}=f_{\mathrm{L}}\left(\hat{\mathrm{x}}_{\mathrm{L}}, \hat{x}_{\mathrm{C} 1}, \hat{v}_{1}, u_{1}\right)+K_{\mathrm{L}} \epsilon_{1} \\
& \hat{x}_{\mathrm{H} 1}=\hat{x}_{\mathbf{H} 2}+K_{\mathrm{H} 1} \epsilon_{1} \\
& \hat{x}_{\mathrm{H} 2}=-\hat{\omega}_{1}{ }^{2} \hat{x}_{\mathrm{H} 1}+K_{\mathrm{H} 2} \epsilon_{1}
\end{aligned}
$$

where $\dot{\hat{x}}_{\mathbf{L}}$ is the estimate of the low frequency state vector of the surge motion, $\hat{x}_{C_{1}}$ is the estimate of the current velocity, $\hat{v}_{1}$ is the estimate of the wind force, $u_{1}$ is the thrust, $\epsilon_{1}$ is the surge coordinate prediction error and $K_{\mathrm{L}}, K_{\mathrm{H} 1}$ and $K_{\mathrm{H}_{2}}$ are filter gains. $\hat{x}_{\mathrm{H} 1}, \hat{x}_{\mathrm{H} 2}$ are the high frequency states and $\boldsymbol{f}_{\mathrm{L}}$ is a vector function, defined by the model eqns. (1) and (2).

If now $\hat{\omega}_{1}$ has a slow variation away from its initial value, the covariance of the innovation process will increase. In order to track the variations in $\omega_{1}$, the following algorithm is applied 


$$
\dot{\hat{\omega}}_{1}=-\frac{k}{2} \frac{\partial}{\partial \hat{\omega}_{1}}\left(\epsilon_{1}{ }^{2}\right)=-k \frac{\partial \epsilon_{1}}{\partial \hat{\omega}_{1}} \epsilon_{1}
$$

This algorithm adjusts $\hat{\omega}_{1}$ in the negative gradient direction of $\epsilon_{1}{ }^{2}$. Because a significant part of $\epsilon_{1}$ stems from measurement and process noise, the constant $k$ has to be given a quite small value so that the noise influence on $\hat{\omega}_{1}$ is kept small. We will now find an expression for $\partial \epsilon_{1} / \partial \hat{\omega}_{1}$ and then discuss the choice of $k$.

$\partial \epsilon_{1} / \partial \hat{\omega}_{1}$ is equal to $-\left(\partial \hat{x}_{H_{1}} / \partial \hat{\omega}_{1}+\partial \hat{x}_{L_{1}} / \partial \hat{\omega}_{1}\right)$. Now, for typical values of $K_{\mathrm{L}}=$ $\left[K_{\mathrm{L} 1}, K_{\mathrm{L} 2}\right]^{\mathrm{T}}, K_{\mathrm{H} 1}, K_{\mathrm{H} 2}$ and $\hat{\omega}_{1}$, it is quite easy to show that $\partial \hat{x}_{\mathrm{L} 1} / \partial \hat{\omega}_{1} \ll \partial \hat{x}_{\mathrm{H} 1} / \partial \hat{\omega}_{1}$. In fact, if $\mathrm{O}\left(\hat{\omega}_{1}\right)=1$, the following relationship holds

$$
\mathrm{o}\left(\frac{\partial \hat{x}_{\mathbf{L} 1}}{\partial \hat{\omega}_{1}}\right)=\mathrm{o}\left(K_{\mathbf{L} 1}\right) \cdot \mathrm{o}\left(\frac{\partial \hat{x}_{\mathbf{H} 1}}{\partial \hat{\omega}_{1}}\right)
$$

where $O($.$) means order of magnitude, and where \mathrm{O}\left(K_{\mathrm{L} 1}\right)$ is typically equal to $10^{-2}$. Hence, by differentiation of eqns. (32) and (33) with respect to $\hat{\omega}_{1}$, we obtain

$$
\begin{aligned}
& \dot{r}=s-K_{\mathrm{H} 1} r \\
& \dot{s}=-\hat{\omega}_{1}{ }^{2} r-2 \hat{\omega}_{1}{ }^{2} \hat{\mathrm{x}}_{\mathrm{H} 1}-K_{\mathrm{H} 2} r
\end{aligned}
$$

where $r=\partial \hat{x}_{\mathrm{H}_{1}} / \partial \hat{\omega}_{1}$ and $s=\partial \hat{x}_{\mathrm{H} 2} / \partial \hat{\omega}_{1}$.

Differentiation of (35) and insertion into (36) yields

$$
\ddot{r}+K_{\mathrm{H} 1} \dot{r}+\left(\hat{\omega}_{1}{ }^{2}+K_{\mathrm{H} 2}\right) r=-2 \hat{\omega}_{1} \hat{x}_{\mathrm{H} 1}
$$

If we how assume that the $H F$-motion estimate is given by

and hence

$$
\hat{x}_{\mathrm{H} 1}=a \sin \hat{\omega}_{1} t
$$

$$
\hat{x}_{\mathrm{H} 2}=a \hat{\omega}_{1} \cos \hat{\omega}_{1} t
$$

the steady state solution to (37) is given by

$$
r=-\frac{\partial \epsilon_{1}}{\partial \hat{\omega}_{1}}=\frac{2 \hat{\omega}_{1}}{\left(K_{\mathrm{H} 1} \hat{\omega}_{1}\right)^{2}+K_{\mathrm{H} 2}{ }^{2}}\left(K_{\mathrm{H} 1} \hat{x}_{\mathrm{H} 2}-K_{\mathrm{H} 2} \hat{x}_{\mathrm{H} 1}\right)
$$

This inserted in eqn. (34), yields the algorithm for tracking of $\omega_{1}$. Now, let us turn to the choice of $k$ in eqn. (34). We introduce the first order approximation

$$
\epsilon_{1}=\epsilon_{10}+\frac{\partial \epsilon_{1}}{\partial \hat{\omega}_{1}} \Delta \omega_{1}
$$

in eqn. (34) where $\epsilon_{10}$ is the innovation signal when $\hat{\omega}_{1}=\omega_{1}$ and where $\Delta \hat{\omega}_{1}=\hat{\omega}_{1}-\omega_{1}$.

This yields if $\omega_{1}$ is assumed to be constant

$$
\Delta \dot{\omega}_{1}=-k\left(\frac{\partial \epsilon_{1}}{\partial \hat{\omega}_{1}}\right)^{2} \Delta \omega_{1}-k \frac{\partial \epsilon_{1}}{\partial \hat{\omega}_{1}} \epsilon_{10}
$$

This yields an approximate time constant for estimation of $\omega_{1}$ given by

$$
T_{\omega}=\frac{1}{k \cdot r^{2}{ }_{\text {efr }}}=\frac{\left(K_{\mathrm{H}_{1}} \hat{\omega}_{1}\right)^{2}+K_{\mathrm{H}_{2}}^{2}}{2 k \hat{\omega}_{1}{ }^{2} \cdot a^{2}}
$$

where $r_{\text {eff }}$ is the effective value of $r=-\partial \epsilon_{1} / \partial \hat{\omega}_{1}$ and $a$ is the amplitude of $\hat{x}_{\mathrm{H} 1}$. If the variance of $\partial \epsilon_{1} / \partial \hat{\omega}_{1}$ is small compared to the variance of $\epsilon_{1}$, the variance of $\hat{\omega}_{1}$ is approximately given by

$$
\operatorname{cov}\left(\hat{\omega}_{1}\right)=\frac{1}{2} k \operatorname{cov}\left(\epsilon_{10}\right)
$$


The structure of the surge and yaw filters are shown in Figs. 4 and 5. The sway filter is identical to the surge filter, except for numerical values of constants, and is not shown separately. The switches B in the filter structures are kept open during the initial phase. The switches are closed when the LF-estimates and the current estimates have converged. Note that the actual implementation of the surge and sway models is slightly more complicated than shown in Fig. 5, because the positions are integrated in earth-fixed coordinates.

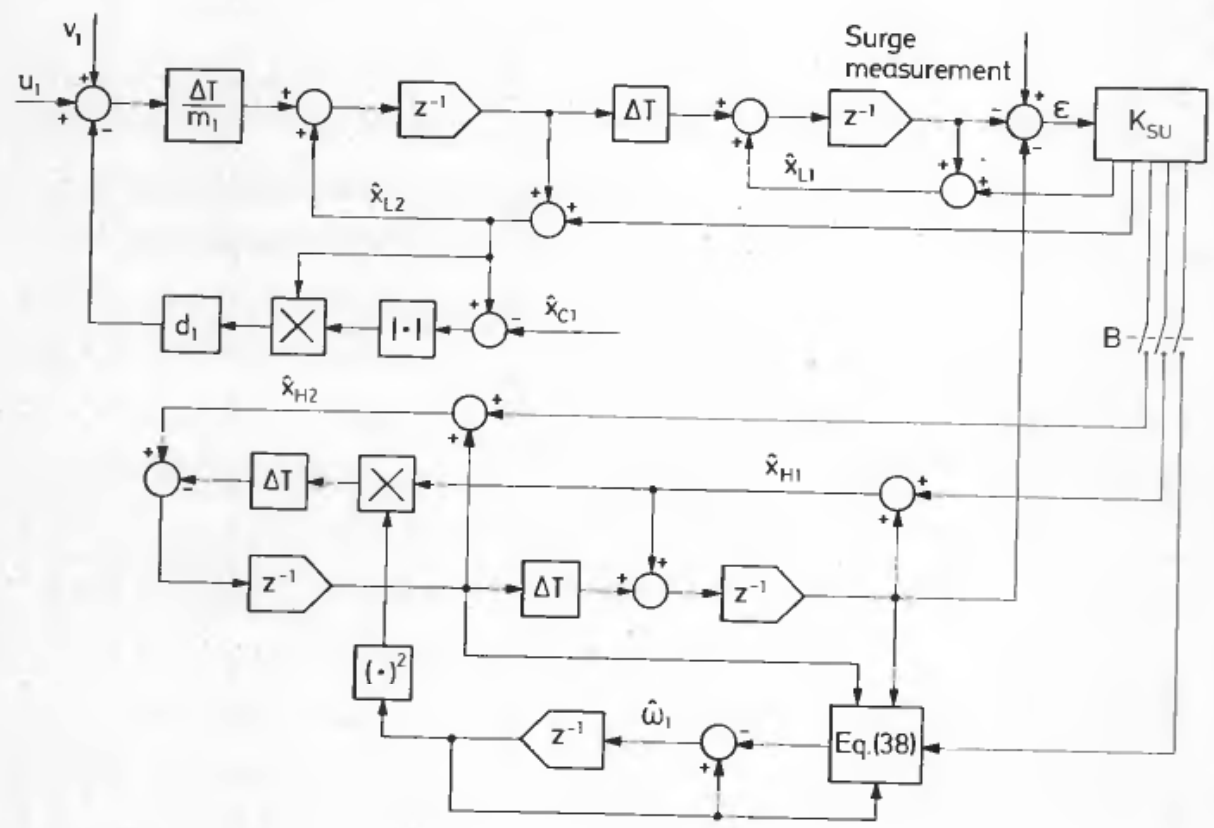

Figure 4. Surge filter structure.

\subsection{The current filter}

The current filter is designed with constant filter gains based on a discretized version of the current model.

As previously indicated the current components are updated by transforming the surge and sway prediction errors to the north oriented system.

The filter on continuous form is given by:

$$
\left[\begin{array}{c}
\hat{x}_{\mathrm{C} 1}{ }^{\mathrm{E}} \\
\hat{x}_{\mathrm{C} 2}{ }^{\mathrm{E}} \\
\hat{x}_{\mathrm{C} 3}{ }^{\mathrm{E}}
\end{array}\right]=K_{\mathrm{C}}\left[\begin{array}{ccc}
\cos \hat{x}_{5} & \sin \hat{x}_{5} & 0 \\
-\sin \hat{x}_{5} & \cos \hat{x}_{5} & 0 \\
0 & 0 & 1
\end{array}\right]\left[\begin{array}{c}
\epsilon_{1} \\
\epsilon_{2} \\
\epsilon_{3}
\end{array}\right]
$$

where $\hat{x}_{5}=\hat{x}_{\mathbf{L} 5}+\hat{x}_{\mathbf{H} 5}$, where $\epsilon_{l}$ are the prediction errors in surge, sway and yaw respectively, and where $K_{\mathrm{C}}$ is a constant, diagonal filter gain matrix. The resulting current filter structure is shown in Fig. 6, where $A$ is defined by eqn. (43). The choice of $K_{\mathrm{C}}$ is discussed later in connection with the control system design. 


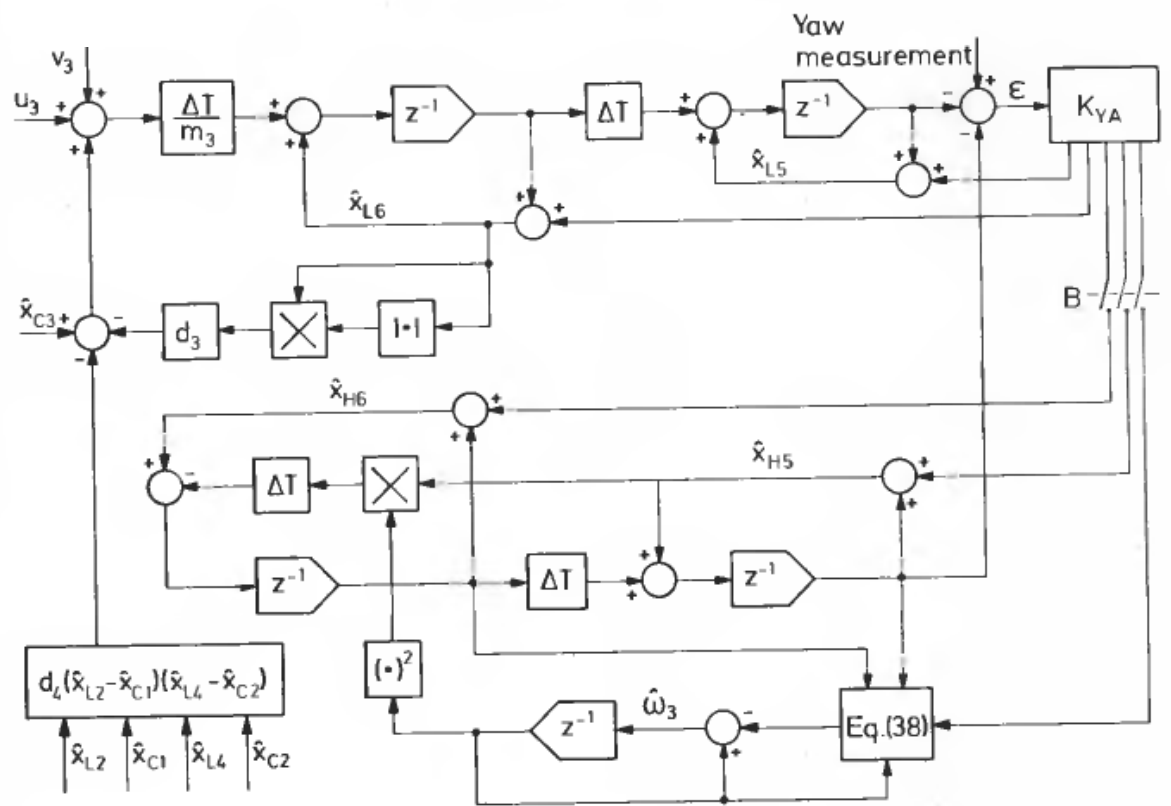

Figure 5. Yaw filter structure.

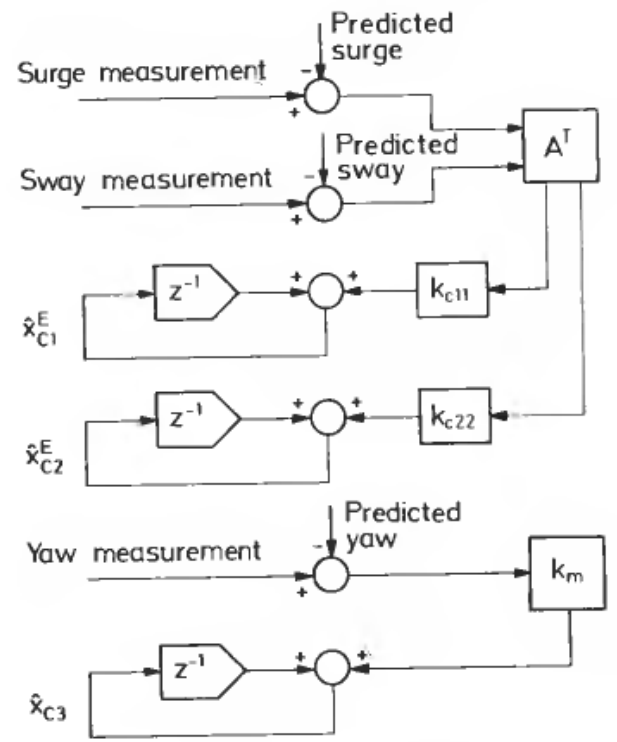

Figure 6. Current filter structure.

\subsection{The wind filter}

As already mentioned, $K_{\mathrm{w}}$ is chosen equal to its steady state value. It is also assumed that the noise of the wind direction model is uncorrelated to the noise of the wind speed model, so that the estimation of the wind speed and the wind direction are decoupled. The discrete wind direction filter is shown in Fig. 7, where $K_{\mathrm{w}}{ }^{4}$ is the wind direction part of $K_{\mathrm{w}}$. Variables marked ' ' are estimates of the corresponding unmarked variables and $\Delta T$ is the sampling interval. 


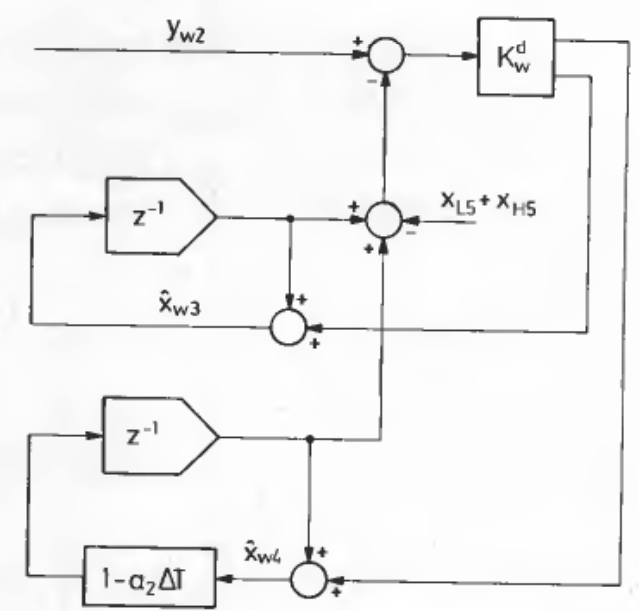

Figure 7. Wind direction filter.

\section{Calibration and error detection}

\subsection{Error detection} are:

Several types of errors may occur in the measurement system. Examples of these

- dead lock of wind sensors due to ice, etc.

-gyro compass failure

- transient failure of hydroacoustic position measurements due to acoustic noise

from thrusters and waves.

Two types of tests are performed on the measurements in order to reject spurious

I measurements and to detect failure in the measurement systems. First, a limit check is

2 applied. If a given measurement is within acceptable limits, a dead lock check is performed. If no dead lock is detected, the measurement is used in the filter algorithm. The limit check of a given measurement is based on a prediction error criterion and works as follows.

7. First, the prediction error is computed. If this error is outside a given error limit, the corresponding measurement signal is disregarded. If the absence of the actual measurement renders the corresponding submodel unobservable, the prediction error limit is increased by a given amount until an acceptable measurement is again obtained. This is illustrated in Fig. 8. If the estimator is without position measurements for too long a time, the estimator will become inactive and will not be activated until a system reset command is given by the operator.

The error detection systems have turned out to work very well. The reason is, of course, that transient errors in the measurement system are not transmitted to the control system which, in the error periods, responds to predicted values of the vessel states and the environmental disturbances.

\subsection{Calibration system}

Once a reference position is established, new measurement systems or new transponders, for use in an already active hydroacoustic measurement system, may be 


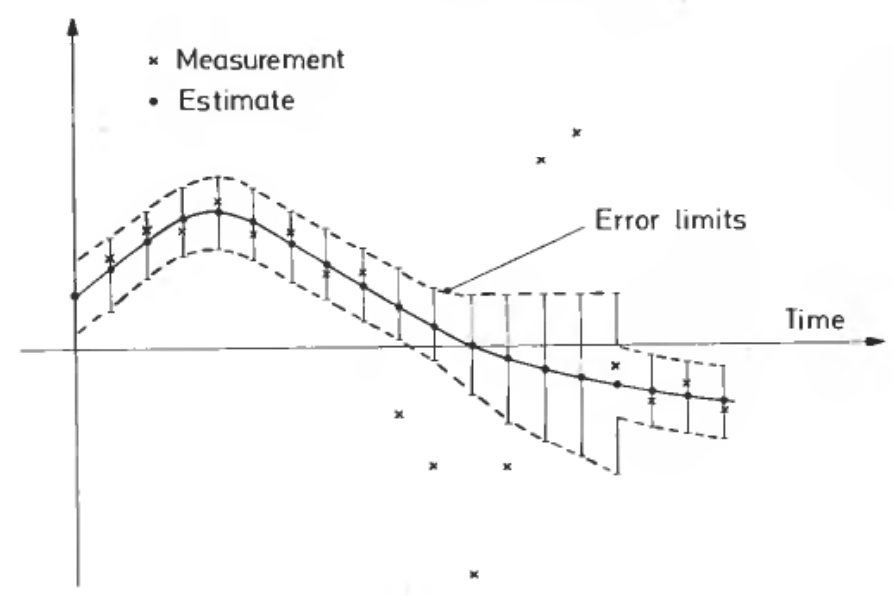

Figure 8. Error detection principle.

calibrated. By calibration, we mean the estimation of the linear translation of the measurements from the calibrating system or transponder relative to the reference position. A measurement from a calibrating system or transponder is used in a simple stochastic approximation algorithm for estimation of the translation coordinates. Several systems may be calibrated simultaneously.

\section{The control system}

The controls are computed from estimates of the environmental states and the deviations between wanted and estimated vessel states. Only the LF-part of the vessel states are used by the control algorithm. This implies that the control signals are almost free from wave-induced, oscillatory components and very little thruster modulation occurs. Feed forward is taken from the wind filter and feedback is taken from the motion and the current estimates. We shall show that the current feedback yields integral action in the control loops.

The total control system structure is shown in Fig. 9. The dynamic properties of this muitivariable control system depend on the Kalman filters, as well as the feedback gain matrices $G$ and $G_{\mathrm{C}}$ and the feed forward gain matrix $G_{\mathrm{w}}$. The matrix $G$ represents the proportional and derivative action of the controller, whereas $G_{\mathrm{C}}$ represents the integral action and $G_{\mathrm{w}}$ the feed forward for wind compensation. We shall now discuss the design of $G, G_{\mathrm{C}}$ and $G_{\mathrm{w}}$.

\subsection{Computation of $G$}

The design of $G$ is based on a quadratic performance criterion and optimal control theory. In order to find $G$, the equations for the vessel behaviour are linearized around zero velocity, zero heading rate and zero current velocity. By transforming the control deviations from earth-fixed to vessel-parallel coordinates, we have obtained three decoupled control problems for surge, sway and yaw, respectively. These control problems are, in principle, identical and we shall only consider the surge control problem. 


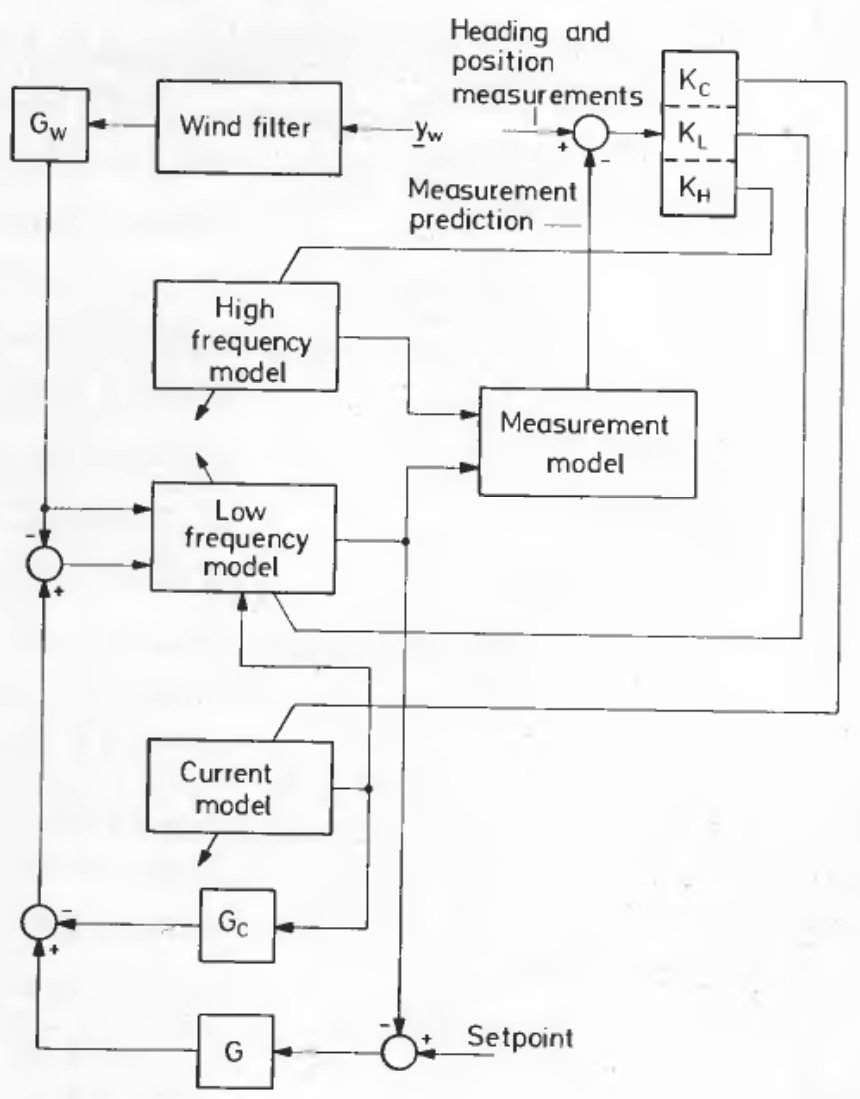

Figure 9. Control system structure.

From eqns. (1) and (2), we have the following linearized surge model if the wind force in neglected:

$$
\begin{aligned}
& \dot{x}_{\mathrm{L} 1}=x_{\mathrm{L} 2} \\
& \dot{x}_{\mathrm{L} 2}=\frac{1}{m_{1}} u_{1}+\eta_{\mathrm{L} 1}
\end{aligned}
$$

We want to find a surge feedback matrix, $G_{\text {su }}$, so that the functional

$$
J=\lim _{T \rightarrow \infty} \frac{1}{T} \int_{0}^{T}\left(q \Delta x_{1}^{2}+p u_{1}^{2}\right) d t
$$

is minimized. $\Delta x_{1}$ is defined as the deviation of $x_{\mathrm{L} 1}$ from its setpoint value. Using standard LQG-theory, we find the optimal gain matrix given by

$$
G_{\mathrm{SU}}=\left[-\sigma,-\sqrt{ }\left(2 m_{1} \sigma\right)\right]
$$

where

$$
\sigma=\sqrt{ }(q / p)
$$


This yields a relative damping of 0.7 . If the relative velocity between the vessel and the water is not zero, (zero is the linearization value), the response of the surge loop with the feedback given by $G_{\mathrm{SU}}$ will be faster and the damping will increase.

$G_{\mathrm{SU}}$ is a submatrix of the block diagonal matrix $G$. The remaining submatrices for sway and yaw are found in exactly the same way.

\subsection{Computation of $G_{\mathrm{W}}$}

$G_{\mathrm{W}}$ is designed so that the output from $G_{\mathrm{w}}$ exactly compensates the predicted wind forces according to the wind model. Hence, we have

$$
u_{\mathrm{w} t}=-\hat{v}_{i}=-f_{i}(\hat{\beta})\left(\hat{x}_{\mathrm{w}_{1}}+\hat{x}_{\mathrm{w}_{2}}\right)^{2}
$$

where $u_{\mathrm{w} i}$ is the contribution to $u_{i}$ from the wind estimates and where $\beta$ is the estimate of $\beta$.

\subsection{Computation of $G_{\mathbf{C}}$}

The feedback via $G_{\mathrm{C}}$ is included to counteract slowly varying environmental curent forces and modelling errors. Now $G$ is designed to take care of rapidly varying external disturbances based on the simple model given by. eqns. (44) and (45). It is, therefore, natural to choose $G_{\mathrm{C}}$ so that the current forces are exactly counterbalanced. If the model were correct, we should therefore, choose (see eqns. (1)-(6))

$$
\begin{aligned}
& u_{\mathrm{C} 1}=d_{1}\left|\hat{x}_{\mathrm{L} 2}-\hat{x}_{\mathrm{C} 1}\right|\left(\hat{x}_{\mathrm{L} 2}-\hat{x}_{\mathrm{C} 1}\right) \\
& u_{\mathrm{C} 2}=d_{2}\left|\hat{x}_{\mathrm{L} 4}-\hat{x}_{\mathrm{C} 2}\right|\left(\hat{x}_{\mathrm{L} 4}-\hat{x}_{\mathrm{C} 2}\right) \\
& u_{\mathrm{C} 3}=d_{4}\left|\hat{x}_{\mathrm{L} 4}-\hat{x}_{\mathrm{C} 2}\right|\left(\hat{x}_{\mathrm{L} 4}-\hat{x}_{\mathrm{C} 2}\right)-\hat{x}_{\mathrm{C} 3}
\end{aligned}
$$

where $u_{\mathrm{C} i}$ is the contribution to $u_{i}$ from the current feedback. Note that $d_{1}, d_{2}$ and $d_{4}$ are functions of the direction of the current relative to the vessel, so that the feedbacks are highly nonlinear.

In a practical situation, there will always be modelling errors involved. However, it is easy to show that, even if modelling errors are present, the expected values of the setpoint deviations will still be zero. Let us, for simplicity, assume that only the surge coordinate is excited, that the current is parallel to the surge axis, and that the total control system is stable. (The proof is easily extended to a general situation). The LF-estimator and the current estimator is then given by

$$
\begin{aligned}
& \hat{x}_{\mathrm{L} 1}=\hat{x}_{\mathrm{L} 2}+k_{\mathrm{SU} 1}\left(y_{1}-\hat{x}_{\mathrm{L} 1}-\hat{x}_{\mathrm{H} 1}\right) \\
& \dot{\hat{x}}_{\mathrm{L} 2}=-\frac{d_{1}}{m_{1}}\left|\hat{x}_{\mathrm{L} 2}-\hat{x}_{\mathrm{C} 1}\right|\left(\hat{x}_{\mathrm{L} 2}-\hat{x}_{\mathrm{C} 1}\right)+\frac{1}{m_{1}}\left(u_{1}+\hat{v}_{1}\right)+k_{\mathrm{SU} 2}\left(y_{1}-\hat{x}_{\mathrm{L} 1}-\hat{x}_{\mathrm{H} 1}\right) \\
& \dot{\hat{x}}_{\mathrm{C} 1}=k_{\mathrm{C} 1}\left(y_{1}-\hat{x}_{\mathrm{L} 1}-\hat{x}_{\mathrm{H} 1}\right)
\end{aligned}
$$

where $k_{\mathrm{SU} 1}, k_{\mathrm{SU} 2}$ and $k_{\mathrm{C} 1}$ are constant filter gains. If we define $\Delta x_{\mathrm{L} 1}=\hat{x}_{\mathrm{L} 1}-x_{\mathrm{L} 1}{ }^{\text {ref }}$ where $x_{\mathrm{L} 1}{ }^{\text {ref }}$ is the position setpoint, (the velocity setpoint is zero), we have

or

$$
u_{1}=G_{\mathrm{SU}}\left[\Delta x_{\mathrm{L} 1}, \hat{x}_{\mathrm{L} 2}\right]^{\mathrm{T}}+u_{\mathrm{C} 1}+u_{\mathrm{w} 1}
$$

$$
u_{1}+\hat{v}_{1}=-\sigma \Delta x_{\mathbf{L} 1}-\sqrt{ }\left(m_{1} \sigma\right) \hat{x}_{\mathbf{L} 2}+d_{1}\left|\hat{x}_{\mathbf{L} 2}-\hat{x}_{\mathbf{C} 1}\right|\left(\hat{x}_{\mathbf{L} 2}-\hat{x}_{\mathrm{C} 1}\right)
$$


This inserted into eqn. (54), yields

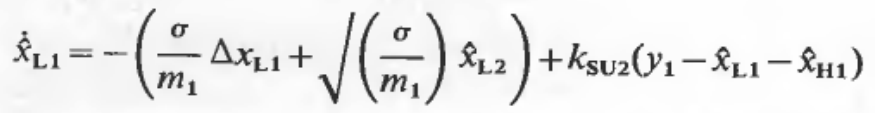

From eqns. (53) and (55), we observe that $E\left(\hat{x}_{L 2}\right)=E\left(y_{1}-\hat{x}_{L 1}-\hat{x}_{H_{1}}\right)=0$. Hence, by using eqn. (57), it is seen that $E\left(\Delta x_{\mathrm{L} 1}\right)=0$. This means that, if the measurement noise has zero mean, the expected value of the surge position will always be equal to its setpoint value.

From this analysis, it is quite obvious that the estimation of $\hat{x}_{\mathrm{C} i}, i=1,2,3$ yields the integral action of the system. The dynamics of this integral action depend heavily upon the choice of the current filter gain matrix $K_{\mathrm{C}}=\operatorname{diag}\left\{k_{\mathrm{C} 1}, k_{\mathrm{C} 2}, k_{\mathrm{C} 3}\right\}$. We shall, therefore, examine the eigenvalues of the total system response introduced by the current feedback. For simplicity, we shall again look at the surge loop and assume that the sway and yaw excitations are zero. The system is linearized around $x_{2}=0$ and $x_{\mathrm{C} 1}=x_{\mathrm{C} 1}{ }^{\text {nom }}$ and we obtain the following linearized representation

$$
\left[\begin{array}{c}
\dot{\hat{x}}_{\mathrm{L} 1} \\
\dot{\hat{x}}_{\mathrm{L} 2} \\
\hdashline \Delta \hat{x}_{\mathrm{L} 1} \\
\Delta \hat{\mathrm{x}}_{\mathrm{L} 2} \\
\hdashline \Delta \hat{\mathrm{x}}_{\mathrm{C} 1}
\end{array}\right]=\left[\begin{array}{c:c:c}
A+B G_{\mathrm{SU}} & K_{\mathrm{SU}} D & 0 \\
\hdashline 0 & A-K_{\mathrm{SU}} D & B g_{\mathrm{C} 1} \\
\hdashline 0 & -k_{\mathrm{C} 1} D & 0
\end{array}\right]\left[\begin{array}{c}
\hat{x}_{\mathbf{L} 1} \\
\hat{x}_{\mathbf{L} 2} \\
\hdashline \Delta \hat{\mathbf{x}}_{\mathbf{L} 1} \\
\Delta \hat{\mathrm{x}}_{\mathbf{L} 2} \\
\hdashline \Delta \hat{x}_{\mathrm{C} 1}
\end{array}\right]+\text { noise }
$$

where

$$
\begin{aligned}
& \Delta \hat{x}=x-\hat{x} \\
& g_{\mathrm{C} 1}=2 d_{1}\left|x_{\mathrm{C} 1}{ }^{\text {nom }}\right| \\
& A=\left[\begin{array}{cc}
0 & 1 \\
0 & -g_{\mathrm{C} 1} / m_{1}
\end{array}\right] \\
& B=\left[\begin{array}{c}
0 \\
1 / m_{1}
\end{array}\right] \\
& D=\left[\begin{array}{ll}
1 & 0
\end{array}\right]
\end{aligned}
$$

The eigenvalues of the total system are seen to be given by the eigenvalues of the submatrices

$$
\left[A+B G_{\mathrm{SU}}\right] \text { and }\left[\begin{array}{c:c}
A-K_{\mathrm{SU}} D & B g_{\mathrm{C} 1} \\
\hdashline-k_{\mathrm{Cl}} D & 0
\end{array}\right]
$$

The eigenvalues of $\left[A+B G_{\mathrm{su}}\right]$ have already been given proper values by the design of $G_{\mathrm{su}}$. The remaining eigenvalues are given by the characteristic equation of the second submatrix

$$
\lambda^{3}+\left(k_{\mathrm{SU} 1}+\frac{g_{\mathrm{C} 1}}{m_{1}}\right) \lambda^{2}+\left(k_{\mathrm{SU} 2}+\frac{g_{\mathrm{C} 1}}{m_{1}} k_{\mathrm{SU} 2}\right) \lambda+\frac{g_{\mathrm{C} 1}}{m_{1}} k_{\mathrm{C} 1}=0
$$


The roots $\lambda_{i}, i=1,2,3$, of this equation satisfy the relation

$$
\lambda_{1} \cdot \lambda_{2} \cdot \lambda_{3}=-k_{\mathrm{C} 1} \frac{g_{\mathrm{C} 1}}{m_{1}}
$$

Now we are only interested in an approximate solution for the eigenvalue, $\lambda_{3}$, introduced by the current feedback. We want to choose $\left|\lambda_{3}\right| \ll\left|\lambda_{1}\right|,\left|\lambda_{2}\right|$. Hence, $\lambda_{1}$ and $\lambda_{2}$ do not change much when the current estimator is included. This yields

$$
\lambda_{1} \cdot \lambda_{2} \approx \operatorname{det}\left(A-K_{\mathrm{SU}} D\right)=k_{\mathrm{SU} 2}+\frac{g_{\mathrm{C} 1}}{m_{1}} k_{\mathrm{SU} 1}
$$

Then, we obtain from eqns. (65) and (66)

$$
\lambda_{3} \approx-\frac{k_{\mathrm{C} 1} g_{\mathrm{C} 1}}{k_{\mathrm{SU} 2} m_{1}+g_{\mathrm{C} 1} k_{\mathrm{SU} 1}}
$$

and by making a proper choice of $\lambda_{3}, k_{\mathrm{C} 1}$ can be computed from

$$
k_{\mathrm{C} 1}=\frac{\left(k_{\mathrm{SU} 2} m_{1}+g_{\mathrm{C} 1} k_{\mathrm{SU} 1}\right)}{g_{\mathrm{C} 1}}\left|\lambda_{3}\right|
$$

A similar formula applies to sway and yaw with $g_{\mathrm{C} 2}$ and $g_{\mathrm{C} 3}$ given by

$$
\begin{aligned}
& g_{\mathrm{C} 2}=2 d_{2}\left|x_{\mathrm{C} 2}{ }^{\text {nom }}\right| \\
& g_{\mathrm{C} 3}=1
\end{aligned}
$$

\section{Simulation experiments}

The development of the estimation and control system was carried out using data for the work platform Seaway Swan, a semisubmersible Aker H3-platform. In this section, we shall give some simulation results where the control system is working against a vessel simulator realized by a computer program. The simulator includes realistic wave excitations, wind and current excitations, measurement noise, etc.

The total mass parameters for the vessel are

$$
\begin{aligned}
& m_{1}=2 \cdot 4 \cdot 10^{7} \mathrm{~kg} \\
& m_{2}=4 \cdot 0 \cdot 10^{7} \mathrm{~kg} \\
& m_{3}=4 \cdot 7 \cdot 10^{10} \mathrm{~kg} / \mathrm{m}^{2}
\end{aligned}
$$

The hydrodynamic parameters are shown in Fig. 10. Based on these parameters and extensive Kalman-filter simulations, the following filter gain matrices are chosen

$$
\begin{aligned}
& K_{\mathrm{SU}}=K_{\mathrm{SW}}=\left[0 \cdot 045,0 \cdot 001,0 \cdot 3,0 \cdot 07, k_{s} \cdot r_{s}\right]^{\mathrm{T}} \\
& K_{\mathrm{YA}}=\left[0 \cdot 145,0 \cdot 004,0 \cdot 7,0 \cdot 15, k_{y} \cdot r_{y}\right]^{\mathrm{T}}
\end{aligned}
$$

where $k_{s}=0.002, k_{y}=0.001$ and $r_{s}$ and $r_{y}$ are given by eqn. (38). For the surge filter we obtain

$$
r_{s}=\frac{2}{0 \cdot 3^{2}+0 \cdot 07^{2}}\left(0 \cdot 3 \hat{x}_{\mathrm{H} 1}-0 \cdot 07 \hat{\omega}_{1} \hat{x}_{\mathrm{H} 2}\right)
$$



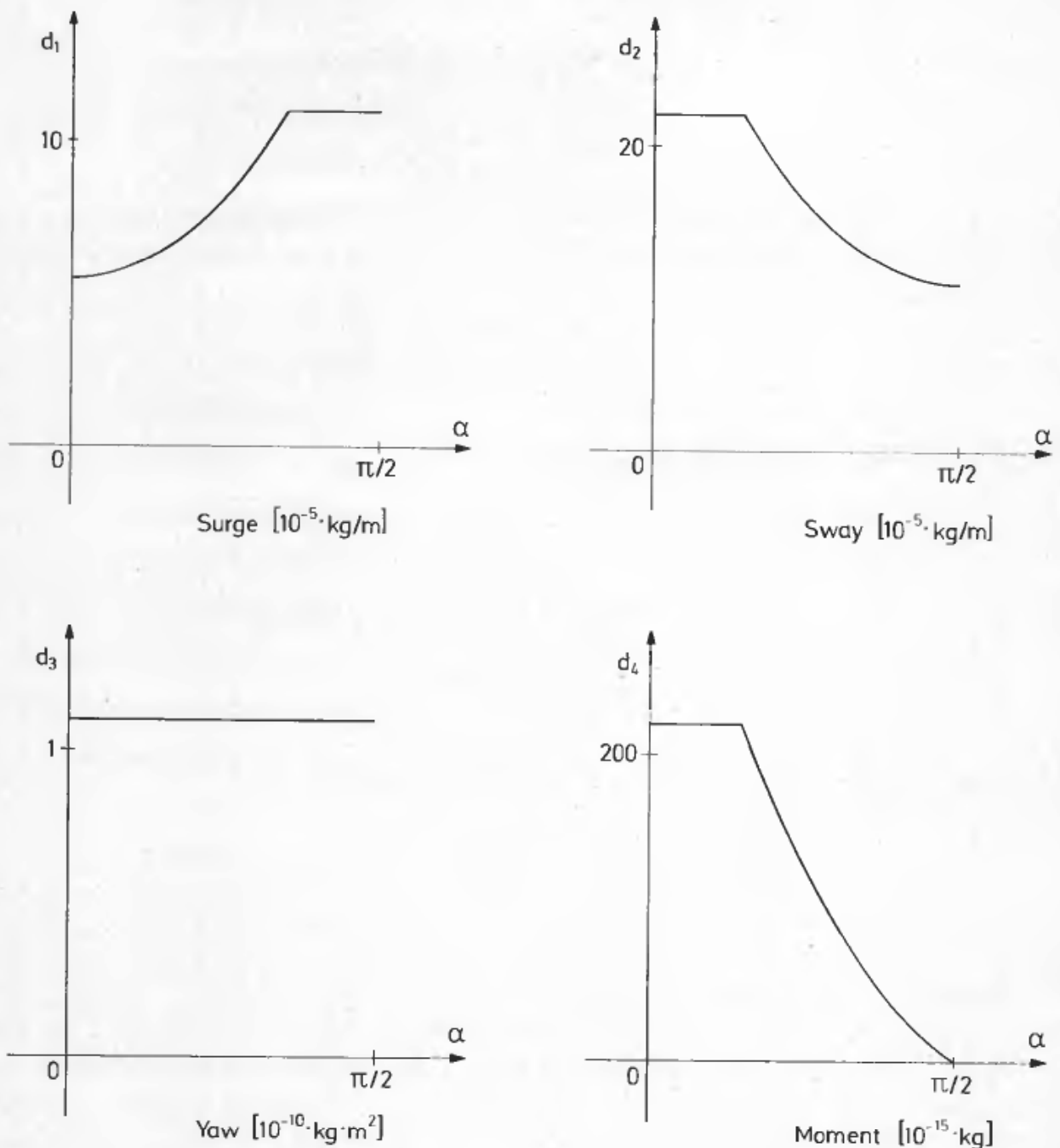

Figure 10. Hydrodynamic drag functions ( $\alpha$ is the angle heading direction and current direction).

The filter is designed using the assumptions of gaussian, white position measurement noise with standard deviation $0.7 \mathrm{~m}$, a gaussian, white heading measurement noise with standard deviation $\mathbf{0 . 2}$ degrees, and a measurement sample rate of 1 second.

In order to test the filter performance, several simulation experiments are carried out. As an example, the estimation of HF and LF motion in the surge coordinate is shown in Fig. 11. In this case, the control system is inactive and the vessel is drifting due to weather and current excitations.

The convergence of the wave frequency estimate $\hat{\omega}_{1}$ is verified in another experiment. Convergence results from different initial estimates of $\hat{\omega}_{1}$ and different measurement noise situations are shown in Fig. 12. The power spectrum of the actual HFmotion of the vessel is shown in Fig. 13. by

From eqn. (41), the time constant for estimation of $\omega_{1}$ is approximately given 


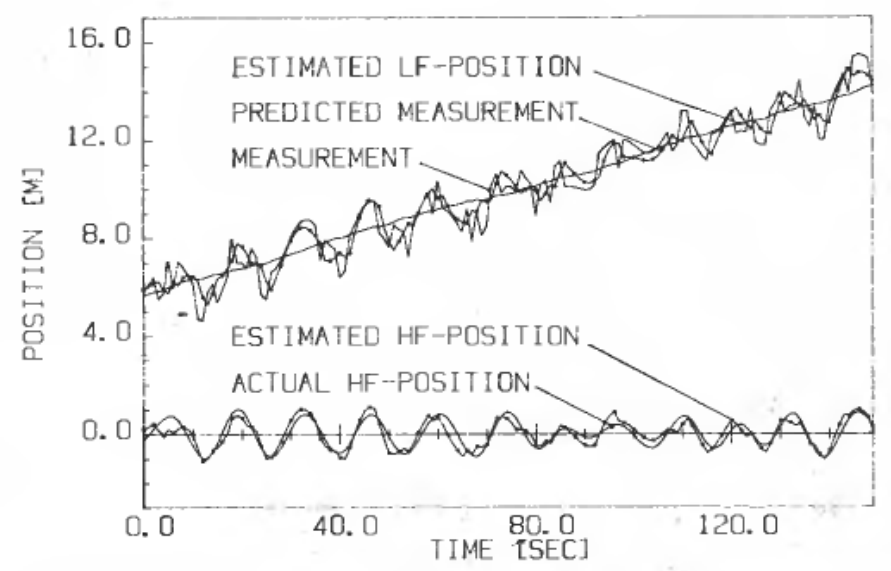

Figure 11 (a). Simulated and estimated position components.

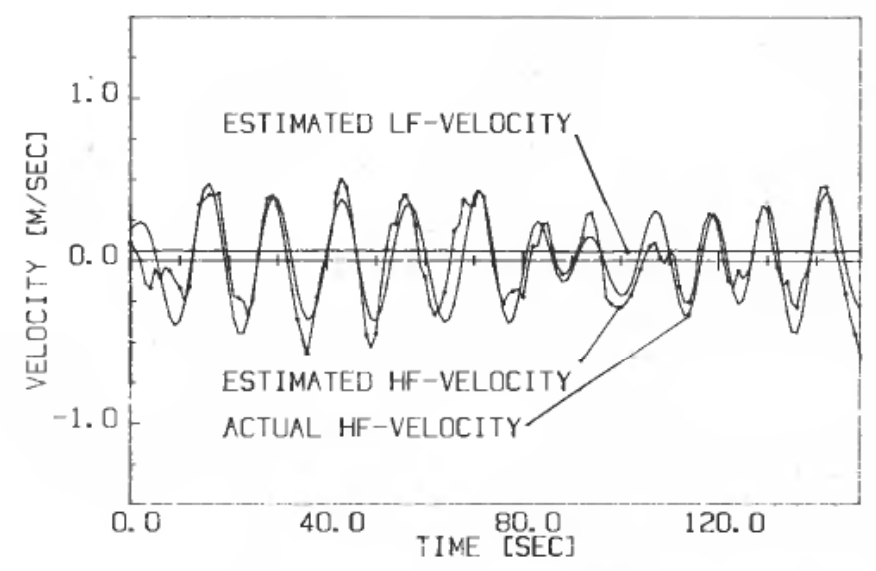

Figure 11 (b). Simulated and estimated HF-velocity.

$$
T_{\omega 1} \approx \frac{16}{\left(\hat{\omega}_{1} a\right)^{2}}
$$

and the standard deviation of $\hat{\omega}_{1}$ is found from eqn. (42) to be approximately given by

$$
\sigma_{\omega 1}=\sigma_{\epsilon} \sqrt{\frac{k_{s}}{2}} \approx 0 \cdot 025
$$

These values of $T_{\omega 1}$ and $\sigma_{\omega 1}$ are seen to agree closely with the simulation results.

The estimation of current is strongly coupled to the control system response. We shall, therefore, discuss the choice of current filter matrix in relation to the control system parameters. The control feedback matrices are chosen to be

$$
\begin{aligned}
G_{\mathrm{SU}} & =\left[3 \cdot 10^{4} \mathrm{~N} / \mathrm{m},-1 \cdot 15 \cdot 10^{6} \mathrm{Ns} / \mathrm{m}\right] \\
G_{\mathbf{S W}} & =\left[-3 \cdot 10^{4} \mathrm{~N} / \mathrm{m},-1 \cdot 55 \cdot 10^{6} \mathrm{Ns} / \mathrm{m}\right] \\
G_{\mathbf{Y A}} & =\left[-2 \cdot 0 \cdot 10^{8} \mathrm{Nm} / \mathrm{rad},-5 \cdot 0 \cdot 10^{9} \mathrm{Nms} / \mathrm{rad}\right]
\end{aligned}
$$




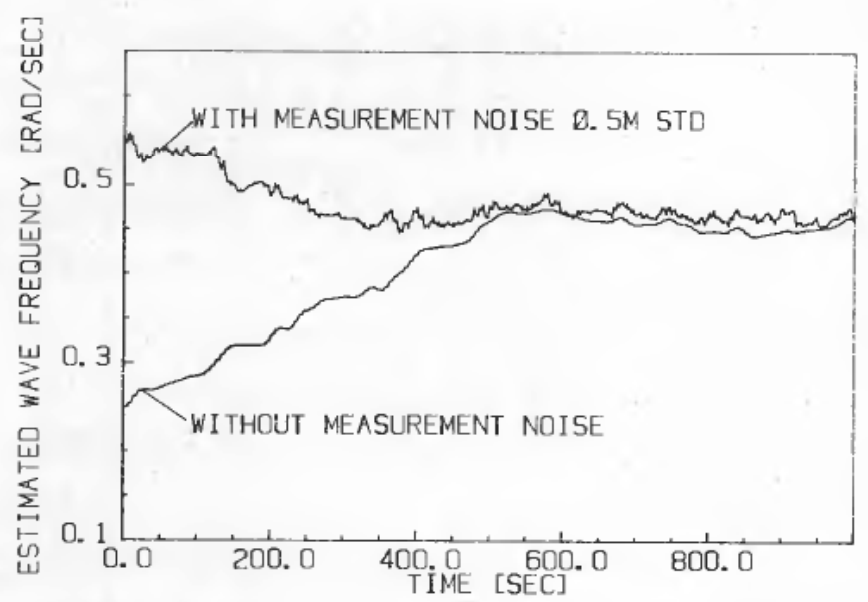

Figure 12. Estimation of HF-motion frequency in surge.

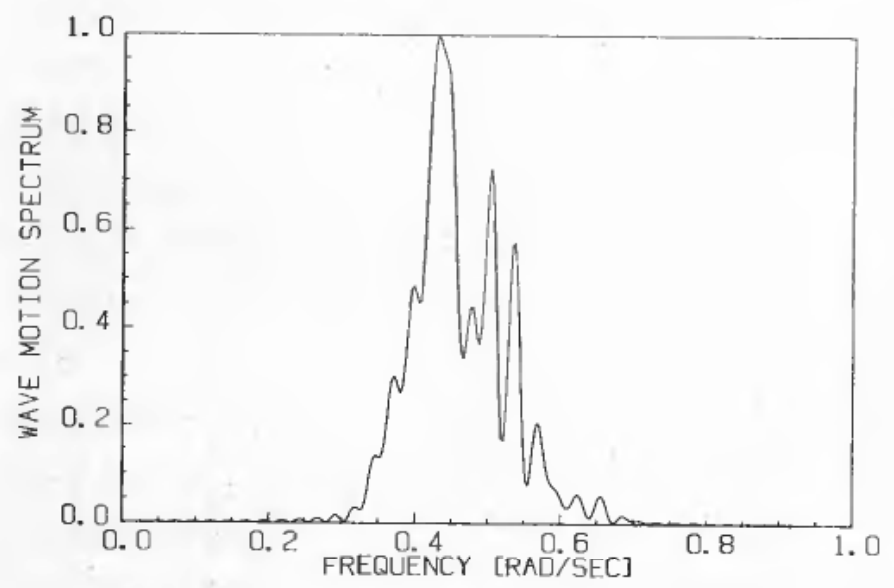

Figure 13. Power spectrum of simulated HF-motion in surge.

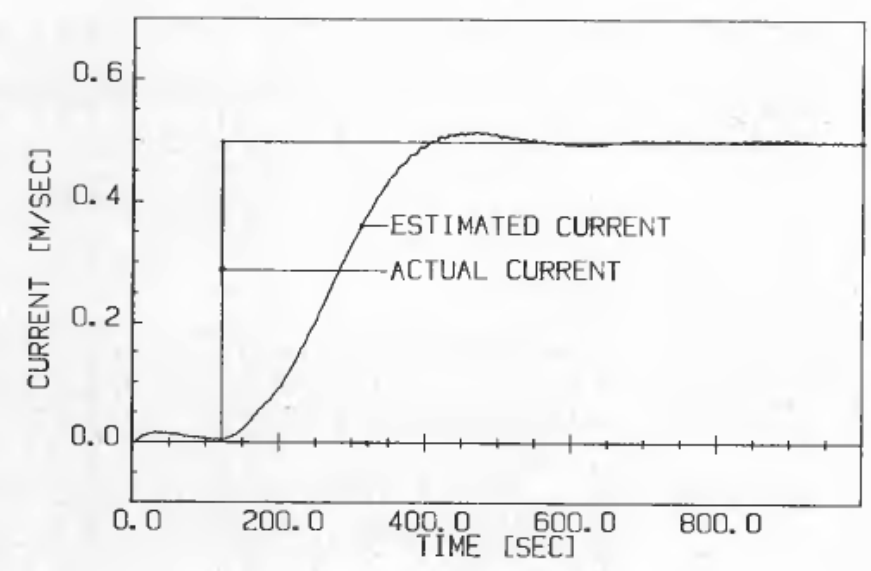

Figure 14. Convergence of current estimator. 
If we examine the surge loop, the eignvalues due to the feedback via $G_{\mathrm{SU}}$ introduce the eigenvalues

$$
\lambda_{1,2}=-0.024 \pm j 0 \cdot 026
$$

Now we assume that the water current is equal to $\hat{x}_{\mathrm{C} 1}$ in the surge direction. From eqn. (67), the eigenvalue coupled to the current estimation loop is given by

$$
\lambda_{3} \approx-\frac{2 d_{1}\left|\hat{x}_{\mathrm{C} 1}\right| \cdot k_{\mathrm{C} 1}}{k_{\mathrm{SU} 2} m_{1}+2 d_{1}\left|\hat{x}_{\mathrm{C} 1}\right| k_{\mathrm{SU} 1}}
$$

With $d_{1}=5 \cdot 10^{5} \mathrm{~kg} / \mathrm{m}, k_{\mathrm{SU} 1}=0 \cdot 045, k_{\mathrm{SU} 2}=0.001$ and $m_{1}=2.4 \cdot 10^{7}$ we obtain

$$
\lambda_{3} \approx-\frac{100 k_{\mathrm{C} 1}\left|\hat{x}_{\mathrm{C} 1}\right|}{2 \cdot 4+4 \cdot 5\left|\hat{x}_{\mathrm{C} 1}\right|}
$$

Choosing $k_{\mathrm{C} 1}=0.001$, we obtain $\lambda_{3}=3 \cdot 6 \cdot 10^{-3}$ for $\hat{x}_{\mathrm{C} 1}=0 \cdot 1 \mathrm{~m} / \mathrm{s}$ and $\lambda_{3}=12 \cdot 0 \cdot 10^{-3}$ for $\hat{x}_{\mathrm{C} 1}=0.6 \mathrm{~m} / \mathrm{s}$. This yields an integral time constant in the range $\sim 100-300$ seconds. $k_{\mathrm{C} 2}$ is chosen to $0 \cdot 001$ and $k_{\mathrm{C} 3}$ is chosen to $2 \cdot 0$.

This agrees closely with simulation results, e.g. as shown in Fig. 14, which shows the convergence of the current estimates after a simulated step change in the water current intensity.

The total control system response to a step change in the position setpoint is shown in Fig. 15. We observe that the heading is kept very nearly constant during the translational motion. This is due to the inclusion of the hydrodynamic interactions between sway and yaw motion in the vessel model.

\section{System implementation}

In the previous sections, we have described the theoretical background and the algorithms worked out for a new generation of DP-systems. In this section and the next, we will describe the practical implementation of this system and discuss the experience gained during almost two years of operation.

A typical system configuration is shown in Fig. 16. The maximum version of the system includes two computers operating in parallel, supervised by a third computer. Only one of the main computers transmits the control signals to the propulsion system. The second computer acts as a hot stand-by computer receiving measurements and processing these by the estimation algorithms. Due to the model-based, integral action produced by the current estimator, the risk of integrator divergence in the hot standby computer, (as experienced in conventional systems), is removed.

The Kongsberg KS 500 computers have a $128 \mathrm{k}$ byte memory and the control and estimation algorithms are written in real-time FORTRAN IV language.

\subsection{Kongsberg DP on $M / V$ Seaway Eagle and $M / V$ Capalonga}

The first version of this new DP-system was developed for the diving and support vessel, Seaway Eagle, operating on the oilfields of the North Sea. Typical operations are maintenance and repair work on the seabed close to platforms and fixed structures. These operations demand the maximum reliability which can be achieved. As a result of this, two reference systems are supplied, a super short baseline hydroacoustic system and a microwave surface reference system. 


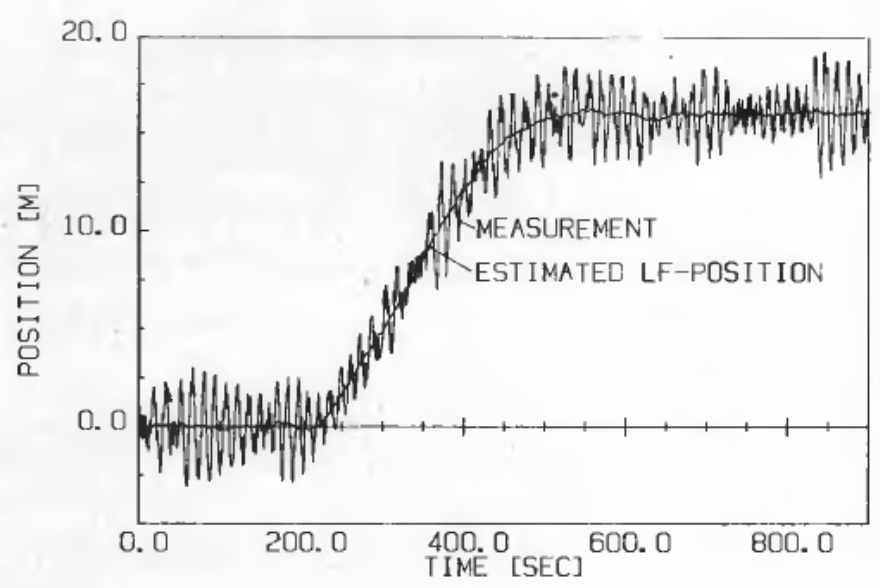

Figure $15(a)$. Simulated measurement and LF-estimate of surge.

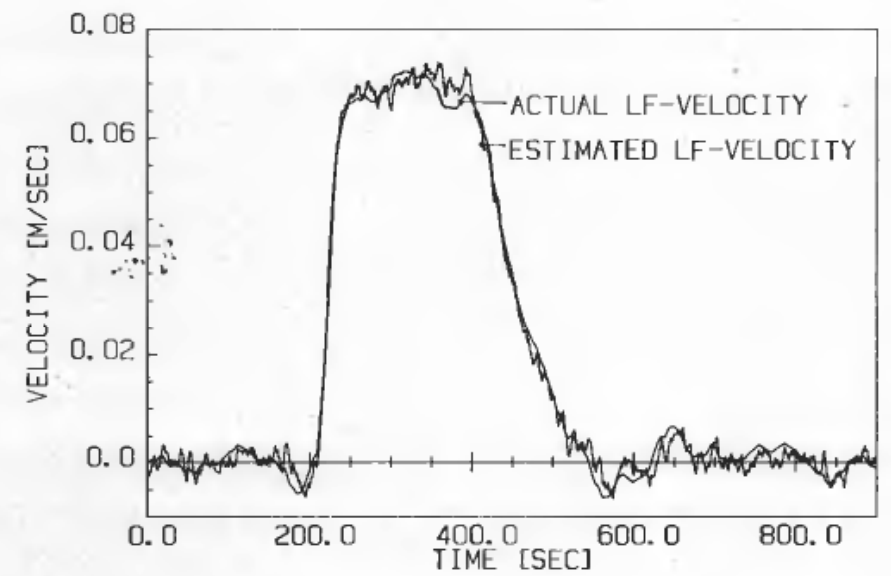

Figure $15(b)$. Simulated and estimated LF-velocity in surge.

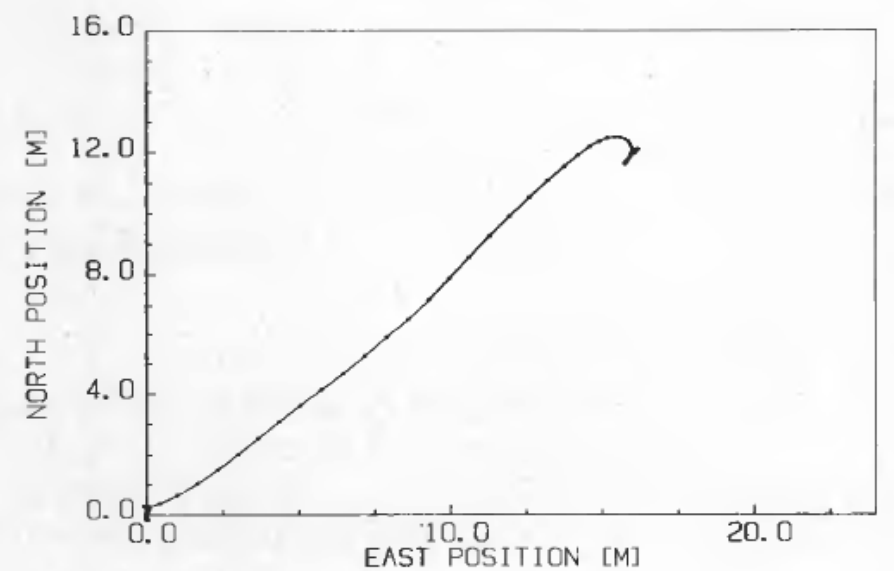

Figure $15(c)$. Position response in earth-fixed coordinates. 


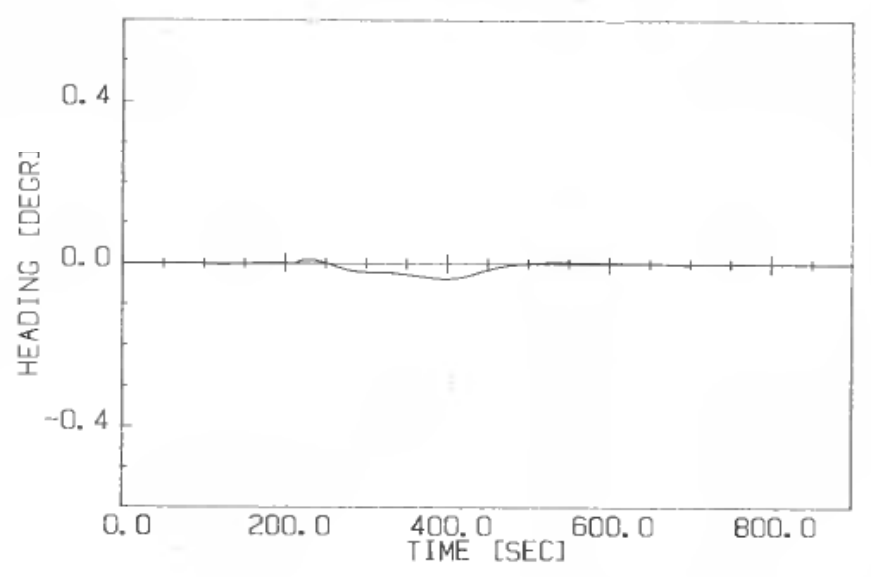

Figure $15(d)$. Heading response.

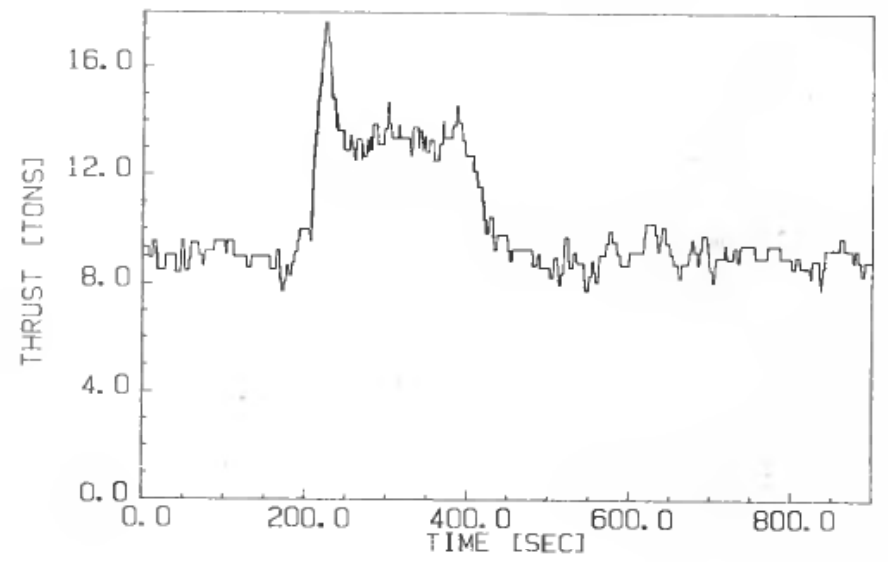

Figure $15(e)$. Applied thrust force in surge.

The different principles of operation and the different transmission media for the two reference systems yield a higher reliability compared to a combination based on identical sensors.

The second installation was carried out for the support vessel $\mathbf{M} / \mathbf{V}$ Capalonga. In addition to the above mentioned sensors, the Capalonga system includes a taut wire sensor. The taut wire measuring system is based on a 0.5 in steel wire rope connected to a depressor weight and exposed to a constant tension, thus indicating position of the vessel via the inclination angles of the wire relative to the vertical.

All practical experience referred to throughout the rest of this paper is from one of these installations, both completed in the year 1977.

\subsection{Simulations and sea trials}

The chosen control concept has several advantages compared to the classical PID control strategy, such as improved noise suppression, more precise dynamic behaviour and improved reliability through prediction and error detection. However, 


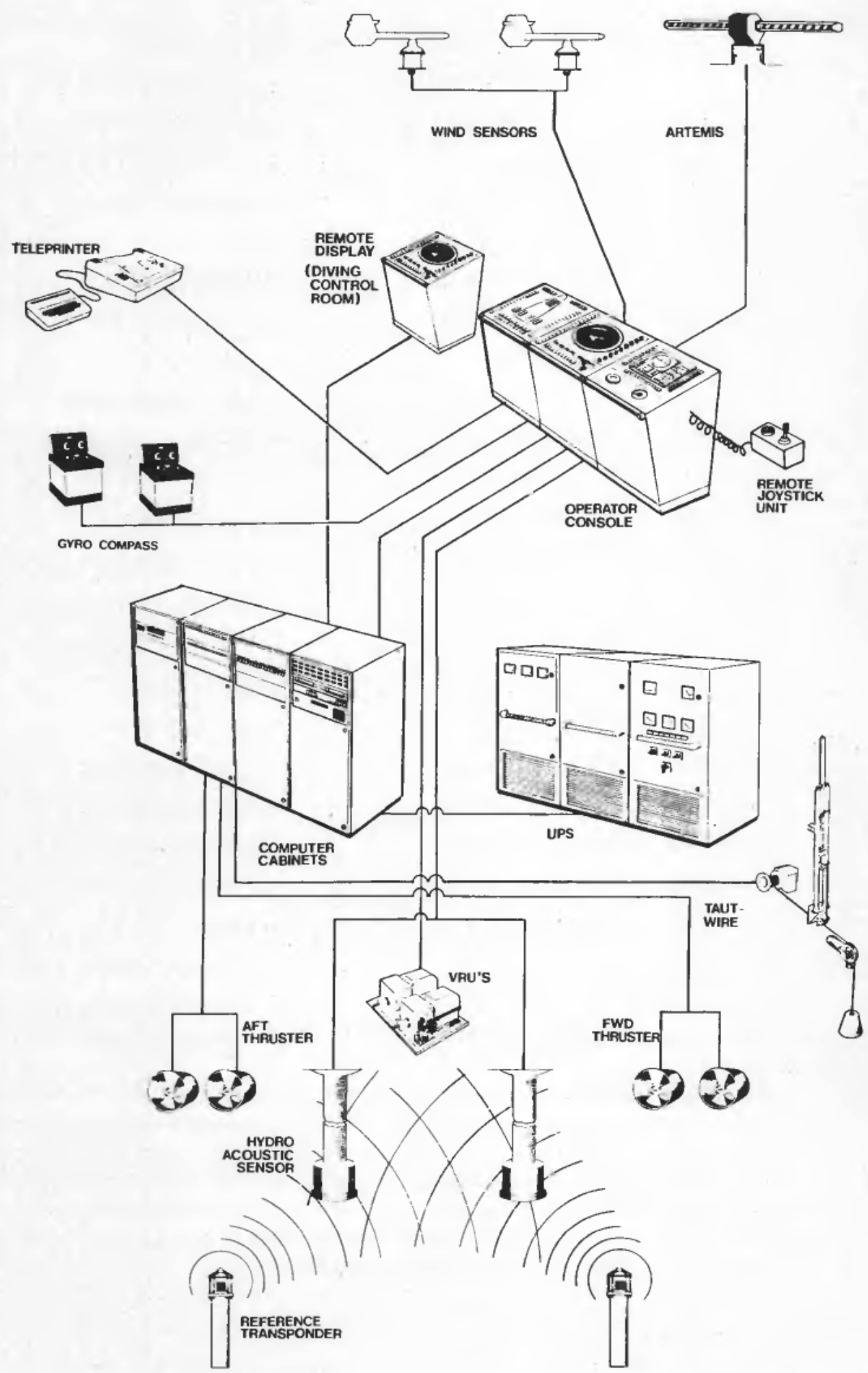

Figure 16. Hardware configuration. 
to achieve these advantages, a substantial amount of computational work has to be carried out prior to installation and testing. One has to compute vessel displacement and added water mass, drag and lift coefficients, wind force parameters, thruster parameters and filter and control system parameters.

Based on computed parameters and coefficients, a software version of the control system is tested and verified against a computer-based vessel simulator in the laboratory. The same simulator is also used for training of operators prior to commissioning of the system.

During a sea trial period all computations and simulation results have to be verified. This is normally done based on simplified parameter estimatation techniques where important parameters are computed from a set of predesigned dynamic experiments.

\section{Operational experience}

A discussion on the properties of a given control system cannot be kept separate from a discussion of the process itself, in this case, the dynamic behaviour of the vessel.

The vessels in question are of about 2000 tons and 5000 tons displacement, respectively. They are equipped with pitch controllable thrusters which, in the case of Capalonga, are azimuth steerable as well. Important parameters of the vessel, when discussing positioning accuracy, are

- thrust capacity

- control accuracy of thrust

- thruster time constants

-seagoing properties of vessel

-interaction forces between thrusters and hull

In the case of these two vessels, the state is not ideal for any of these factors but both have satisfactory performance.

We shall now discuss the system performance, mainly with respect to noise suppression, control properties and prediction and error detection capabilities.

\subsection{Noise suppression}

As could be expected, the Kalman filter works extremely well. This has made it possible to utilize reference systems not previously used for dynamic positioning systems, such as the PULSE 8 navigation system. A hydroacoustic reference system used in unfavourable conditions and in deep water often yields measurement signals with considerable disturbances. The efficient filtering of these signals has significantly reduced the thruster control excitations from measurement noise. Figure 17 shows the innovation signal from the surge loop using hydroacoustics. The actual water depth is $100 \mathrm{~m}$ and the vessel is constantly increasing its distance from the transponder, while at the time $1000 \mathrm{sec}$ the vessel is about 100 meters away from the transponder. We observe that the measurement noise increases with increasing distance. An innovation signal equal to zero (see Fig. 17), indicates an erroneous signal, detected either by signal check in the hydroacoustic receiver or by the prediction error check in the Kalman filter.

The standard deviation of the innovation signal in the worst case conditions of Fig. 17 equals about $1.5 \mathrm{~m}$. This introduces disturbances in position and velocity 


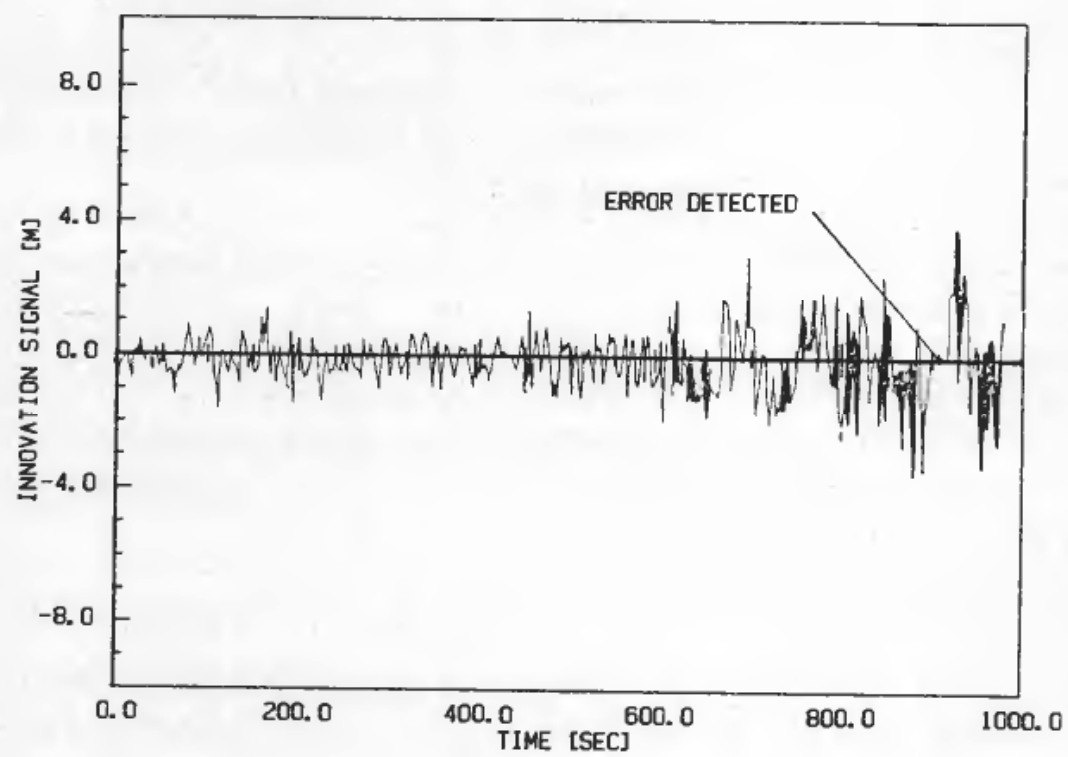

Figure 17. Innovation signal of surge measurement.

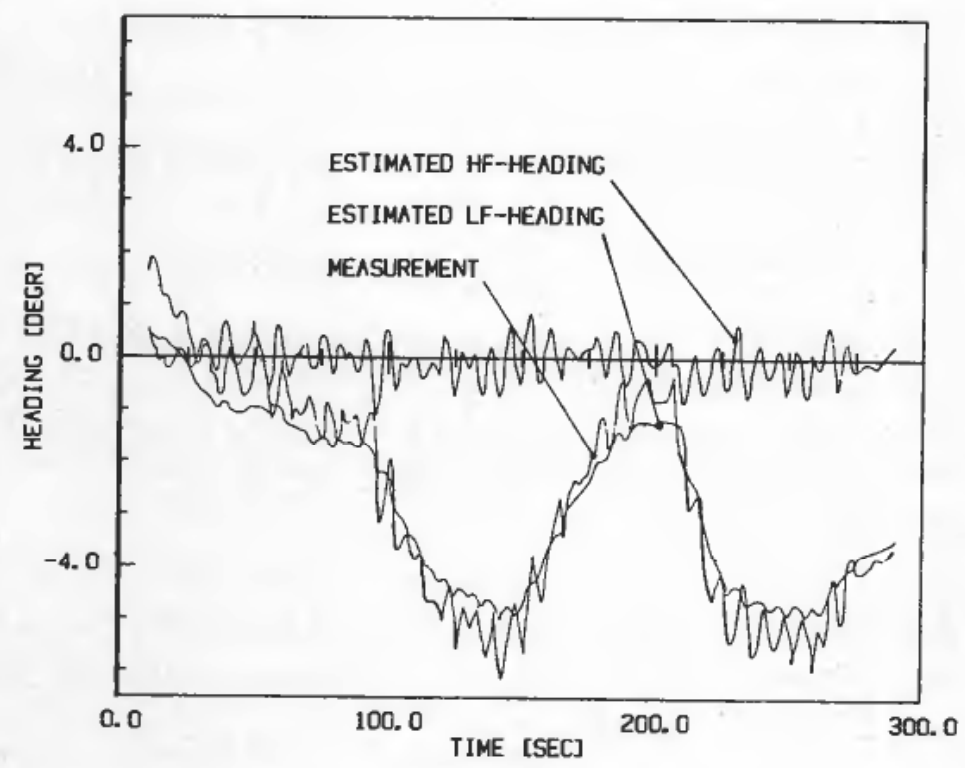

Figure 18. Measured yaw motion together with LF- and HF-estimates.

estimates of about $0.1 \mathrm{~m}$ and $0.03 \mathrm{~m} / \mathrm{s}$, respectively, interpreted as standard deviations. For the vessel Seaway Eagle, with control loop parameters

$$
G=\left(-2 \text { tons } / \mathrm{m},-30 \text { tons } / \mathrm{ms}^{-1}\right)
$$

we get thruster excitations of approximately $1 \cdot 1$ tons. This corresponds to about $10 \%$ of available thrust in the lateral direction. The deadband is normally set to $5 \%$ of full scale and will, therefore, reduce the number of thruster setpoint updates significantly, even under extremely unfavourable reference system conditions. 
The wave filter described in section 3 also turns out to be highly successful. Figure 18 shows a sequence of heading measurements together with the low frequency and the high frequency estimate during severe weather conditions on board the Seaway Eagle. The significant waveheight is above $5 \mathrm{~m}$ and the mean wave period is $7 \cdot 5 \mathrm{sec}$. We observe that the heading low frequency estimate follows the measurement with insignificant phase lag and with a wave suppression of 8 to $10 \mathrm{~dB}$.

\subsection{Optimal control}

One main advantage of the control concept is that the estimators are also running in manual control mode. This is valid of course, only if a measurement system is

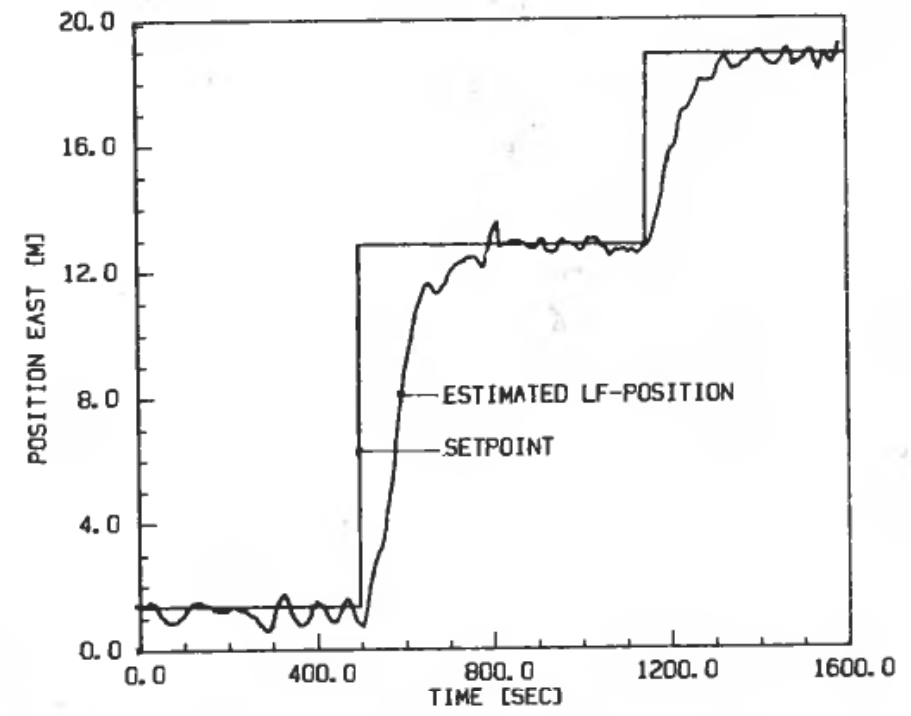

Figure $19(a)$. Vessel response in east position.

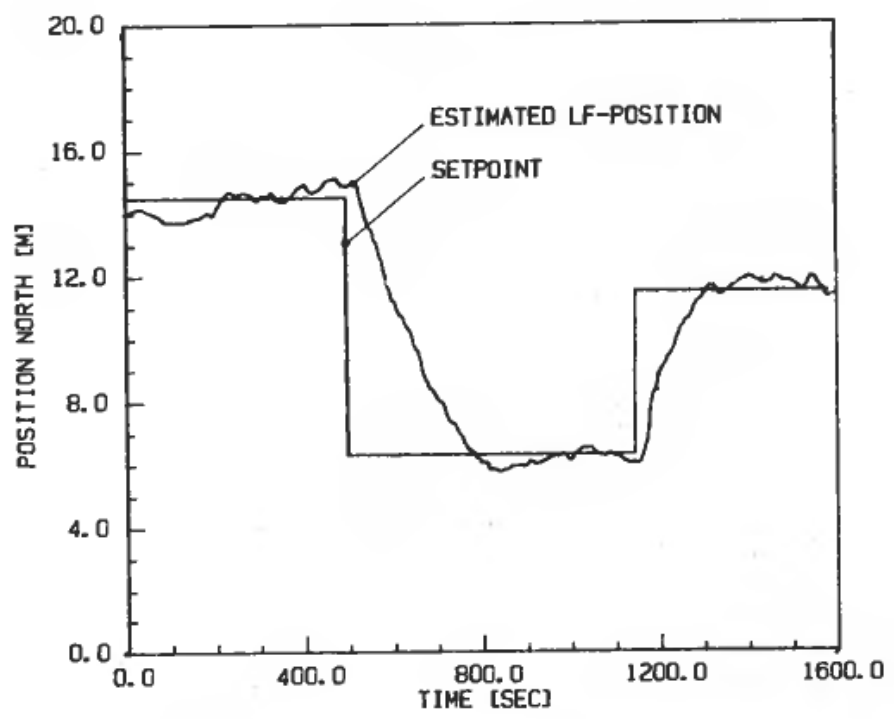

Figure $19(b)$. Vessel response in north position. 
operative. The obvious effect is the possibility to enter the automatic control mode without a transient deviation due to the integral action, as the water current is already known by the current estimator. This is of high value when operating close to a fixed structure or a vessel, as only small deviations can be allowed in these situations.

The operational experience shows a smooth and precise control of the rotational and translational motion of the vessel.

As an example, Fig. 19 shows the reaction to setpoint changes in the north and east coordinates of the vessel Seaway Eagle. A motion trace of the Seaway Eagle, during station keeping in moderate weather conditions, is shown in Fig. 20. Figure 21 shows a typical control signal sequence to the propeller pitch servo during station keeping in moderate weather conditions.

The following emperical expression for maximum control deviation may be formulated

$$
d=(0 \cdot 5 S+2 f \cdot w h+0 \cdot 01 w s+0 \cdot 5)[\text { meters] }
$$

where $S$ is the standard deviation of the position measurement, (sampling rate 1 second), $f$ is the amplitude of the vessel wave motion, wh is the significant waveheight and $w s$ is the wind speed.

As an example, a system based on a supershort baseline hydroacoustic position reference system with accuracy $1 \%$ of water depth at $100 \mathrm{~m}, f=0 \cdot 3, w h=3 \mathrm{~m}$ and $w s=15 \mathrm{~m} / \mathrm{s}$, yields $d=3 \mathrm{~m}$ corresponding to $3 \%$ of water depth.

\subsection{Prediction and error detection}

Measurement blocking is quite often encountered during operation, especially when using hydroacoustic systems. In recent years it has become more common to use two reference systems simultaneously. Some vessel owners and operators even specify this. However, using only one reference system, it is of considerable importance that the system reacts properly when measurements are blocked in any way. The estimator based control concept described here is very well suited to handle such

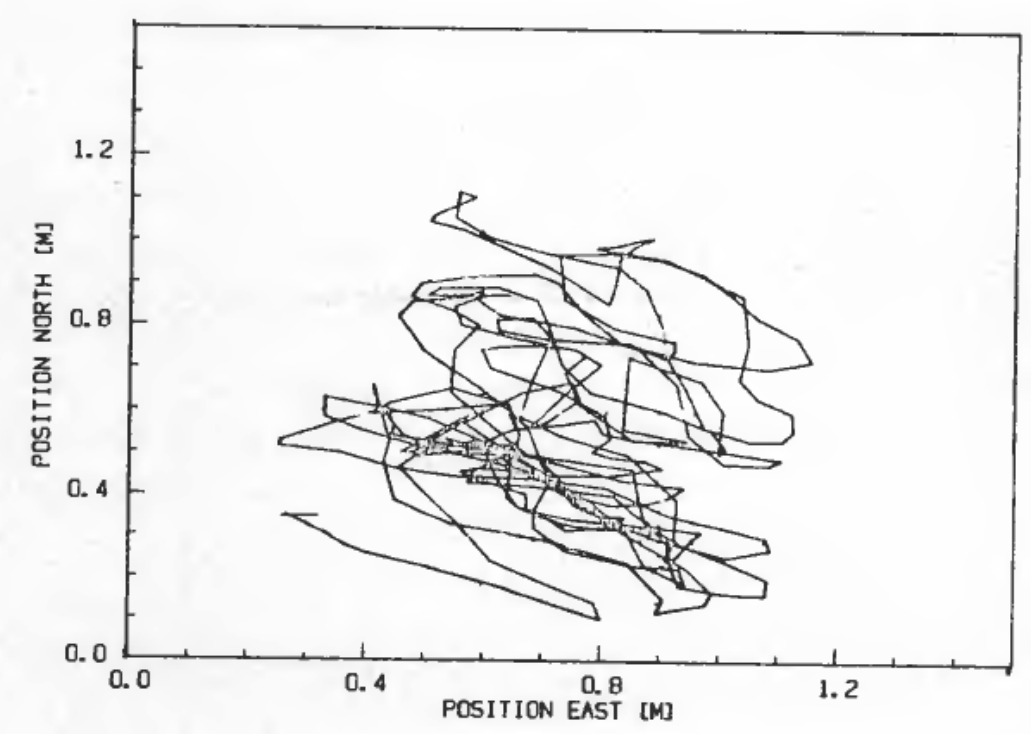

Figure 20. Motion trace of M/V Seaway Eagle during station keeping. 


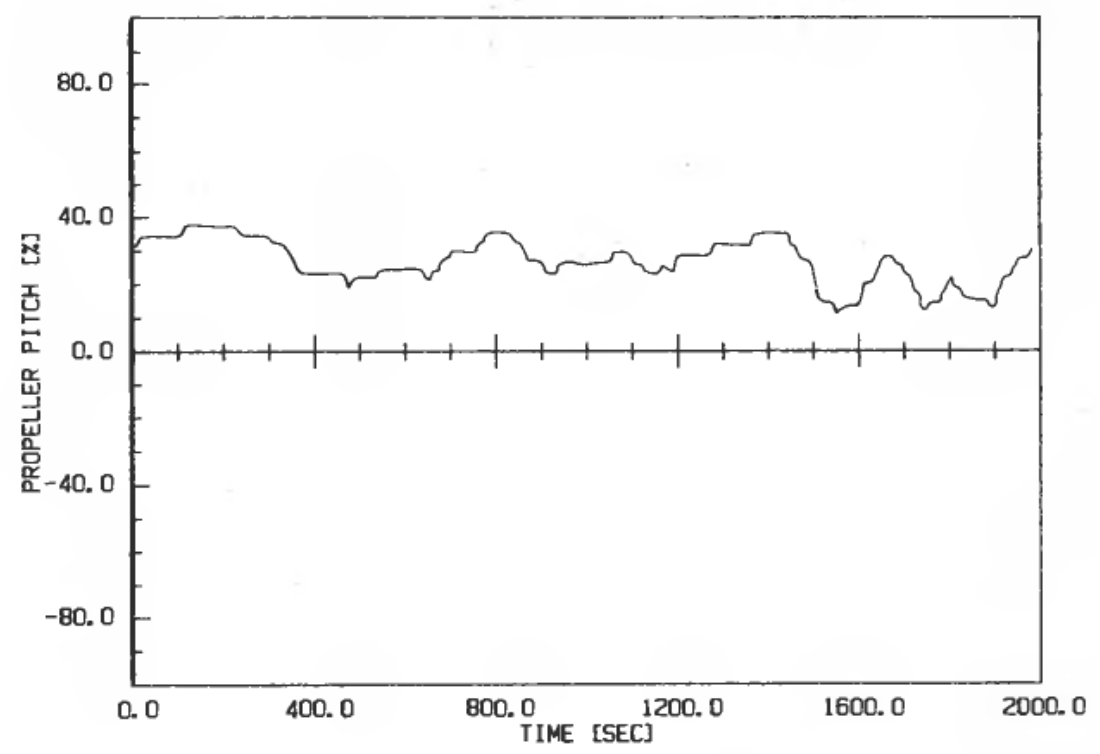

Figure 21. Typical control single sequence.

situations. Loss of measurements is disregarded by the estimator and no state update will take place. Ballistic prediction will, therefore, be used as input for the force demand computations. The wind feed forward through the estimator will of course, still be active. The accuracy of the vessel position estimates will, slowly decrease in such a situation. Since current changes are very slow compared to the relevant time scale, the accuracy will mainly depend on the wave height and the wave characteristics of the vessel.

Through practical experience, we may say that, in a five minute period, a vessel of the Seaway Eagle type will have a drift off of normally less than ten meters. A large vessel will perform better than a smaller one in this respect, due to the greater mass and the smaller wave response.

\section{Conclusion}

Modern control theory has been successfully applied to the dynamic positioning problem. Operational experience has shown that the resulting system behaves in accordance with the expectations or even better, in some aspects. The price to be paid is a more complicated software, increased computer capacity requirements and increased engineering effort in the specific system design. Recent experience has shown that the market is willing to pay for the extra advantages.

\section{REFERENCES}

Balchen, J. G., Jenssen, N. A., and SÆLud, S., (1976). Dynamic Positioning Using Kalman Filtering and Optimal Control Theory. IFAC/IFIP Symposium on Automation in Offshore Oil Field Operation. (North-Holland Publishing Company).

Jazwinski, A. H., (1970). Stochastic Process and Filtering Theory. (New York: Academic Press).

Sargent, J. S., and Cowgill, P. N., (1976). Design Considerations for Dynamically Positioned Utility Vessels. Offshore Technology Conference, Dallas, U.S.A. 